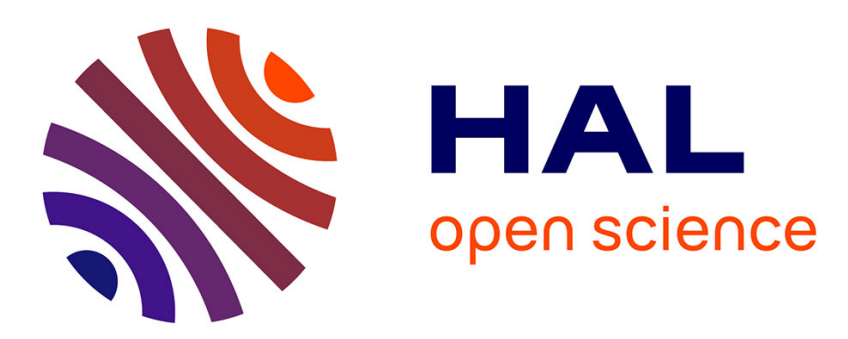

\title{
Towards wall functions for the prediction of solute segregation in plane front directional solidification
}

M. Chatelain, S. Rhouzlane, V. Botton, M. Albaric, D. Henry, S. Millet, D. Pelletier, J.-P. Garandet

\section{- To cite this version:}

M. Chatelain, S. Rhouzlane, V. Botton, M. Albaric, D. Henry, et al.. Towards wall functions for the prediction of solute segregation in plane front directional solidification. Journal of Crystal Growth, 2017, 475, pp.55-69. 10.1016/j.jcrysgro.2017.05.019 . cea-01212315v3

HAL Id: cea-01212315

https://hal-cea.archives-ouvertes.fr/cea-01212315v3

Submitted on 24 Feb 2021

HAL is a multi-disciplinary open access archive for the deposit and dissemination of scientific research documents, whether they are published or not. The documents may come from teaching and research institutions in France or abroad, or from public or private research centers.
L'archive ouverte pluridisciplinaire HAL, est destinée au dépôt et à la diffusion de documents scientifiques de niveau recherche, publiés ou non, émanant des établissements d'enseignement et de recherche français ou étrangers, des laboratoires publics ou privés. 


\title{
Towards wall functions for the prediction of solute segregation in plane front directional solidification
}

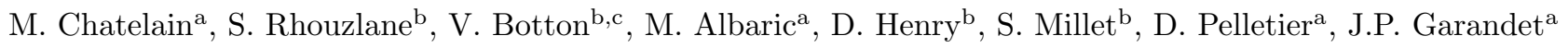 \\ ${ }^{a}$ Univ. Grenoble Alpes, INES, F-73375 Le Bourget du Lac, France. \\ CEA, LITEN, Department of Solar Technologies, F-73375 Le Bourget du Lac, France. \\ ${ }^{b}$ Laboratoire de Mécanique des Fluides et d'Acoustique, CNRS/Université de Lyon, \\ Ecole Centrale de Lyon/Université Lyon 1/INSA Lyon, ECL, 36 Avenue Guy de Collongue, 69134 Ecully Cedex, France. \\ ${ }^{c} I N S A$ Euromed, Université Euro-Méditerranéenne de Fès, route de Meknes BP 51, 30000 Fez, Morocco.
}

\begin{abstract}
The present paper focuses on solute segregation occurring in directional solidification processes with sharp solid/ liquid interface, like silicon crystal growth. A major difficulty for the simulation of such processes is their inherently multi-scale nature: the impurity segregation problem is controlled at the solute boundary layer scale (micrometers) while the thermal problem is ruled at the crucible scale (meters). The thickness of the solute boundary layer is controlled by the convection regime and requires a specific refinement of the mesh of numerical models. In order to improve numerical simulations, wall functions describing solute boundary layers for convecto-diffusive regimes are derived from a scaling analysis. The aim of these wall functions is to obtain segregation profiles from purely thermo-hydrodynamic simulations, which do not require solute boundary layer refinement at the solid/liquid interface. Regarding industrial applications, various stirring techniques can be used to enhance segregation, leading to fully turbulent flows in the melt. In this context, the scaling analysis is further improved by taking into account the turbulent solute transport. The solute boundary layers predicted by the analytical model are compared to those obtained by transient segregation simulations in a canonical 2D lid driven cavity configuration for validation purposes. Convective regimes ranging from laminar to fully turbulent are considered. Growth rate and molecular diffusivity influences are also investigated. Then, a procedure to predict concentration fields in the solid phase from a hydrodynamic simulation of the solidification process is proposed. This procedure is based on the analytical wall functions and on solute mass conservation. It only uses wall shear-stress profiles at the solidification front as input data. The 2D analytical concentration fields are directly compared to the results of the complete simulation of segregation in the lid driven cavity configuration. Finally, an additional output from the analytical model is also presented. We put in light the correlation between different species convecto-diffusive behaviour; we use it to propose an estimation method for the segregation parameters of various chemical species knowing segregation parameters of one specific species.
\end{abstract}

Keywords: directional solidification, segregation, boundary layer, turbulent transport, scaling analysis

\section{Introduction}

The present work is related to solidification from the melt processes with sharp solid/liquid interface, like silicon crystal growth. In such processes solute segregation represents an important issue regarding material quality. For instance, in the field of photovoltaic silicon production, the control of dopant concentrations and the removal of metallic impurities are important parameters regarding solar cells efficiency [1-3]. The numerical simulation of heat, momentum and solute transport in the melt is a powerful tool for process optimizations [4-6]. Nevertheless, the variety of involved length scales is a drawback for the study of industrial configurations. First of all, a precise description of the furnace thermal conditions is mandatory for a realistic description of the crystallization process, including growth rate variations and interface curvature. Heat transfers between the main components of the furnace must be computed, resulting in characteristic dimensions in the range of meters for industrial scale furnaces [7-9]. On the other hand, the description of the solute boundary layer at the solidification front requires a very thin discretization. For silicon crystal growth under natural convection, the solute boundary layer thickness is in the order of $1 \mathrm{~mm}$ (assuming a molecular diffusivity $D \sim 10^{-8} \mathrm{~m}^{2} / \mathrm{s}$ and an interface velocity $V_{I} \sim 10 \mu \mathrm{m} / \mathrm{s}$ ). The use of stirring systems to enhance segregation leads to even thinner solute boundary layers with possibly fully turbulent flows [10-14]. Therefore, there is a real interest in the development of coupled methods, with heat and momentum 
transport simulations on one side and solute transport scaling analysis on the other side. This simplification of the problem remains reasonable as far as dilute alloys are concerned, solute concentrations remaining sufficiently low to have negligible influence on heat and momentum transport.

In their fundamental work on solute segregation, Burton et al. [15] have established that a key parameter of the segregation problem is the thickness of the solute boundary layer at the solid/liquid interface, which is controlled by molecular diffusion and convective transport in the melt. A definition of the solute boundary layer thickness $\delta$ has been proposed by Wilson [16]. This definition proved to be an efficient way to determine the effective segregation coefficient $k_{\text {eff } f}$ from a convecto-diffusive parameter $\Delta$. Recently, Garandet et al. [17] introduced an analytical model of the solute boundary layer. This model is derived from a scaling analysis of the solute transport equation and predicts the convecto-diffusive parameter from the wall shear-stress at the solid/liquid interface. In their study, a steady-state 2D lid driven cavity configuration is used as a reference case for segregation under various convective regimes. Then, Kaddeche et al. [18] tested this model on an horizontal Bridgman configuration and compared it with experimental and numerical results. Both studies highlight the ability of the model to predict the mean segregation regime from an averaged value of the wall shear-stress obtained from a hydrodynamic simulation of the melt convection flow. This model was also tested in a transient regime with a 2D lid driven cavity flow (Chatelain et al. [19]). This study shows that the analytical model provides a good estimate of the lateral segregations using local values of wall shear-stress at the solid/liquid interface. The model can also describe transient variations of the segregation regime for low frequency perturbations of the convection regime and solidification rate, in the limits of the quasi-steady assumption used for the scaling analysis of the solute boundary layer.

The aim of this paper is to demonstrate that an analytical formulation of the solute boundary layer, derived from a scaling analysis, can provide a meaningful description of segregations occurring in directional solidification processes. This approach leads to the definition of solute wall functions describing solute boundary layers for convecto-diffusive regimes, up to fully turbulent flows. Section 2 is dedicated to the formulation and validation of such solute wall functions. The original model proposed by Garandet et al. [17] is revised, in order to account for turbulent transport inside the solute boundary layer. Solute boundary layers obtained from these wall functions are compared to numerical results of segregation in the transient lid driven cavity configuration, which proved to be a meaningful parametric reference case [19]. In section 3, the developed analytical model is used to retrieve segregation profiles from hydrodynamic simulations. An iterative procedure based on solute mass conservation is proposed to access solute repartition in the solid. This procedure is applied to the 2D lid driven cavity configuration and the analytical concentration fields are directly compared to those obtained numerically. Finally, section 4 presents an application of the model for the study of different species segregation. A correlation between the segregation parameters of the different species is derived from the solute boundary layer model.

\section{Turbulent transport in solute boundary layers}

\subsection{Solute boundary layer analytical model}

We consider a quasi 1D directional solidification process. A weakly concentrated liquid metallic blend or semiconductor is progressively solidified. The plane solidification front progresses in the $z$ direction. Let us recall that a jump in impurity (or solute) concentration at the solid/liquid interface is imposed between concentrations on the

liquid side, $C_{L}^{I}$, and the solid side, $C_{S}^{I}$, since the impurity solubility is different in these two phases. Very often a smaller solubility is observed in the solid than in the liquid, leading to a thermodynamic segregation coefficient, $k_{0}=C_{S}^{I} / C_{L}^{I}$, smaller than one. The consequence is that, while the solidification progresses, solute is rejected from the solid phase towards the liquid phase, which leads to the formation of a solute boundary layer in the liquid in the vicinity of the front. Burton et al. [15] explain that solute incorporation in the solid depends on the solute boundary layer at the solidification front. Wilson [16] proposed the following definition of the solute boundary layer thickness:

$$
\delta=\frac{C_{L}^{I}-C_{L}^{\infty}}{-\left(\partial C_{L} / \partial z\right)_{I}},
$$

where $C_{L}^{I}$ and $C_{L}^{\infty}$ stand for solute mass fraction in the liquid at the solid/liquid interface and outside the solute boundary layer, respectively. Assuming negligible diffusion in the solid, solute conservation at the interface is given by:

$$
-\left.D \frac{\partial C_{L}}{\partial z}\right|_{I}=V_{I}\left(1-k_{0}\right) C_{L}^{I},
$$


with $D$ the solute molecular diffusivity in the liquid $\left(\mathrm{m}^{2} / \mathrm{s}\right), V_{I}$ the interface velocity $(\mathrm{m} / \mathrm{s})$ and $k_{0}$ the thermodynamic segregation coefficient. The effective segregation coefficient is then defined by:

$$
k_{e f f}=\frac{k_{0} C_{L}^{I}}{C_{L}^{\infty}}=\frac{k_{0}}{1-\left(1-k_{0}\right) \Delta},
$$

where the convecto-diffusive parameter $\Delta$ represents the normalized solute boundary layer thickness:

$$
\Delta=\frac{\delta V_{I}}{D}=\frac{C_{L}^{I}-C_{L}^{\infty}}{C_{L}^{I}\left(1-k_{0}\right)}
$$

since the solute boundary layer thickness for a purely diffusive regime is given by the ratio $D / V_{I}$.

The analytical model proposed by Garandet et al. [17] describes the solute boundary layer in convecto-diffusive regimes. It is derived from a scaling analysis of the solute transport equation in the liquid phase. At first, the analysis was presented for the lid-driven cavity configuration, but it can easily be transposed to other configurations. We shall consider here the general case of a boundary layer flow developing over a plane solidification front. This configuration is presented in figure 1. A boundary-layer flow develops over the solid/liquid interface, from an impingement point (on the right side) to a separation point (on the left side). The characteristic length over which the boundary layer flow takes place is denoted $l_{c}$, and the vertical velocity gradient at the interface leads to a wall shear-stress $\tau=\mu(\partial u / \partial z)_{z=0}$, with $u$ the velocity component parallel to the interface $(\mathrm{m} / \mathrm{s})$ and $\mu$ the liquid dynamic viscosity (Pa.s).

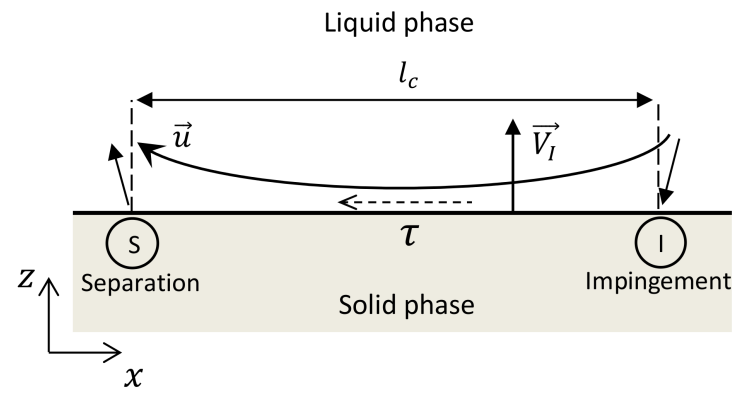

Figure 1: Boundary layer flow configuration (impingement and separation points, characteristic length $l_{c}$, interface velocity $V_{I}$, schematic flow pattern and interface shear-stress $\tau$ ).

As explained in former studies [17, 20], the scaling analysis of the solute transport equation leads to a simplified equation for $\delta$ :

$$
\frac{D}{\delta}=V_{I}-w(\delta)
$$

where $w(\delta)$ stands for the convection velocity normal to the interface, at the edge of the solute boundary layer. As proposed by Garandet et al. [17], this term can be estimated from the wall shear-stress at the solid/liquid interface $\tau$, assuming a linear velocity profile inside the solute boundary layer for the tangential component $u$. Equation (5) can then be rewritten in the dimensionless form:

$$
1-\Delta-B \Delta^{3}=0 .
$$

With this formulation, the convecto-diffusive parameter $\Delta$ is computed from a unique dimensionless parameter denoted $B$ and defined by:

$$
B=\frac{\tau D^{2}}{V_{I}^{3} \mu l_{c}} .
$$

In the following, the analytical solution of equation (6) proposed by Garandet et al. [17] is referred to as $\Delta_{t h}$. Figure 2 presents the evolution of $\Delta_{t h}$ as a function of $B$. The transition between diffusive $\left(\Delta_{t h}=1\right)$ and convective regimes $\left(\Delta_{t h} \approx B^{-1 / 3}\right)$ occurs for $B \approx 1$. To perform this analysis, Garandet et al. [17] assume negligible turbulence effects within the solute boundary layer. For all convective regimes, the solute boundary layer is supposed to remain thinner than the viscous sublayer of the velocity boundary layer (which leads to the assumption of a linear velocity 
profile in the solute boundary layer). This assumption is, however, questionable when fully developed turbulent flows are involved. Numerical simulations in quasi-steady [17] and transient [19] configurations highlight the limits of this assumption and show that $\Delta_{t h}$ significantly overestimates the convecto-diffusive parameter $\Delta$ for moderate to high values of $B$. Moreover, the value of $B$ above which discrepancies occur is seen to depend on the considered impurity and on the growth conditions, through the value of $D$ and $V_{I}$.

The thickness of the viscous sublayer $\delta_{v s l}$ is classically normalized by means of the inner length scale $\nu / u_{\tau}$, where $\nu=\mu / \rho$ stands for the kinematic viscosity $\left(\mathrm{m}^{2} / \mathrm{s}\right)$ and $u_{\tau}=\sqrt{\tau / \rho}$ represents the friction velocity $(\mathrm{m} / \mathrm{s}), \rho$ being the liquid density $\left(\mathrm{kg} / \mathrm{m}^{3}\right)$. The dimensionless thickness of the viscous sublayer $\delta_{v s l}^{+}$, is then expressed by the following relation:

$$
\delta_{v s l}^{+}=\delta_{v s l} \frac{u_{\tau}}{\nu} .
$$

In the literature [21], $\delta_{v s l}^{+}$is considered to be of the order of 5 (in wall units). In order to compare $\delta_{v s l}$ to the thickness of the solute boundary layer, it is useful to normalize by $D / V_{I}$. Introducing $D / V_{I}$ and $B$ in equation (8), we can express the normalized viscous sublayer thickness $\Delta_{v s l}$ by the following relation:

$$
\Delta_{v s l}=\delta_{v s l}\left(V_{I} / D\right)=\delta_{v s l}^{+}\left(B R e_{V_{I}}\right)^{-1 / 2},
$$

where the parameter $\operatorname{Re}_{V_{I}}=\left(V_{I} l_{c}\right) / \nu$ is a Reynolds number based on the interface velocity. Using equation (9), viscous sublayer and solute boundary layer thicknesses can be compared in a dimensionless form. The evolution of $\Delta_{v s l}$ with $B$ is also given in figure 2. Here liquid silicon properties are used with $\rho=2550 \mathrm{~kg} / \mathrm{m}^{3}$ and $\mu=7.5 \times 10^{-4}$ Pa.s. The interface velocity is set at $V_{I}=10 \mu \mathrm{m} / \mathrm{s}$, the characteristic length $l_{c}$ is set at $0.2 \mathrm{~m}$ and the dimensionless viscous sublayer thickness is $\delta_{v s l}^{+}=5$. For moderate convective regimes, $\Delta_{t h}$ remains smaller than $\Delta_{v s l}$. But when $B$ increases, $\Delta_{t h}$ tends to $B^{-1 / 3}$ whereas $\Delta_{v s l}$ is proportional to $B^{-1 / 2}$. A critical regime is necessarily found with a viscous sublayer getting as thin as the solute boundary layer. This critical regime is not universal but depends on configuration parameters. Beyond this limit, turbulent transport should not be neglected within the solute boundary layer.

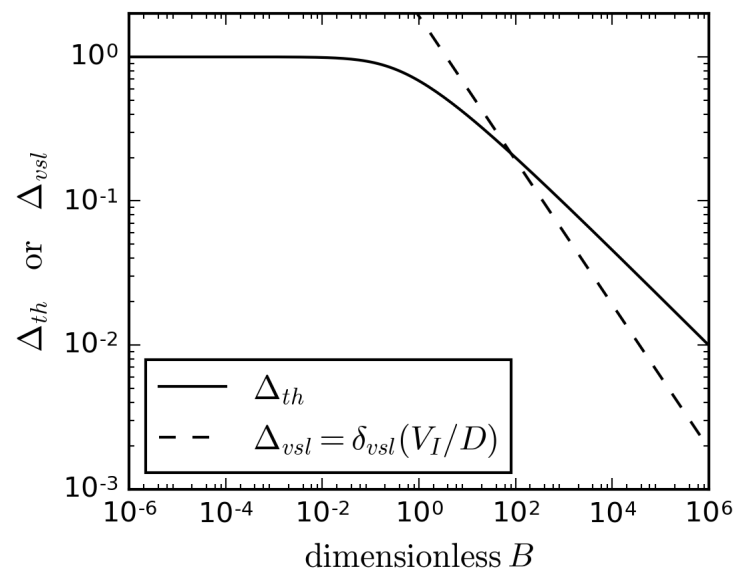

Figure 2: Evolution of $\Delta_{t h}$ and $\Delta_{v s l}=\delta_{v s l}\left(V_{I} / D\right)$ with $B$. To define $\Delta_{v s l}$ liquid silicon properties are used $\left(\rho=2550 \mathrm{~kg} / \mathrm{m}^{3}\right.$ and $\left.\mu=7.5 \times 10^{-4} \mathrm{~Pa} . \mathrm{s}\right)$, the interface velocity is set at $V_{I}=10 \mu \mathrm{m} / \mathrm{s}$, the characteristic length $l_{c}$ is set at $0.2 \mathrm{~m}$ and $\delta_{v s l}^{+}$is set at 5 .

At this point, we should highlight that a recent study from Altenberend et al. [22] was also dedicated to the influence of turbulent boundary layers on solute segregation during silicon solidification. This theoretical, numerical and experimental study focuses on a 1D parallel flow (Couette configuration). It confirms that, when high growth rates are achieved, a high stirring velocity and turbulent transport in the solute boundary layer is required for an efficient segregation. A theoretical model based on turbulent boundary layers properties is proposed and leads to the definition of a threshold value of the stirring velocity to ensure an efficient segregation.

In order to study high convective regimes, generated by stirring systems for instance, the original scaling analysis proposed by Garandet et al. [17] should be revised to account for turbulent transport in the solute boundary layer. To describe turbulent transport we rely on a standard turbulence model [21] based on the Reynolds decomposition and the definition of an eddy viscosity $\nu_{t}\left(\mathrm{~m}^{2} / \mathrm{s}\right)$. A turbulent diffusivity $D_{t}$ can be defined through a turbulent 
$S c h m i d t$ number $S c_{t}=\nu_{t} / D_{t}$. In this study, the turbulent $S c h m i d t$ number is assumed to be constant. Reference studies $[23,24]$ provide a reasonable value of $S c_{t}=0.7$. It is worth mentioning, however, that the definition of $S c_{t}$ is still an open question. Some studies suggest that it should be a function of several parameters, including the distance from the wall $[25,26]$. In the frame of our work, the solute transport equation in the liquid is:

$$
\frac{\partial C_{L}}{\partial t}+(\vec{u} . \nabla) C_{L}=\nabla \cdot\left[\left(D+D_{t}\right) \nabla C_{L}\right]
$$

$C_{L}$ and $\vec{u}$ stand for the solute mass fraction in the liquid and the mean velocity field $(\mathrm{m} / \mathrm{s})$, respectively. Here the word "mean" refers to the RANS approach, turbulent fluctuations influence being accounted for by $D_{t}$. Considering a one dimensional steady-state configuration, in a frame moving with the interface and with $z$ pointing from the solid to the liquid, equation (10) becomes:

$$
D \frac{\partial^{2} C_{L}}{\partial z^{2}}+\frac{\partial}{\partial z}\left(D_{t} \frac{\partial C_{L}}{\partial z}\right)+\left(V_{I}-w\right) \frac{\partial C_{L}}{\partial z}=0
$$

where $w$ stands for the mean convection velocity normal to the interface. Thereafter, we should follow the scaling procedure proposed by Garandet et al. [20]. But further information about the turbulent diffusion term $D_{t}\left(\partial C_{L} / \partial z\right)$ is first required. On one hand, the turbulent diffusivity $D_{t}$ is null at the interface and increases with $z$. On the other hand, the vertical concentration gradient $\partial C_{L} / \partial z$ is negative in the solute boundary layer. The product $D_{t}\left(\partial C_{L} / \partial z\right)$ then decreases from 0 to reach a negative value at $z=\delta$. In a scaling analysis framework, the derivative $\partial\left[D_{t}\left(\partial C_{L} / \partial z\right)\right] / \partial z$ can then be considered as negative inside the solute boundary layer. This negative sign is consistent since turbulent diffusion is introduced to describe turbulent convective transport of the solute away from the interface.

To perform the scaling of equation (11) we consider that the solute concentration variation in the solute boundary layer is given by $\Delta c=C_{L}^{I}-C_{L}^{\infty}$. Using Wilson's definition of the solute boundary layer thickness $\delta$, in an order of magnitude approach equation (11) becomes:

$$
D \frac{\Delta c}{\delta^{2}}-D_{t}(\delta) \frac{\Delta c}{\delta^{2}}-\left(V_{I}-w(\delta)\right) \frac{\Delta c}{\delta}=0,
$$

or, after simplification by $\Delta c / \delta$ :

$$
\frac{D}{\delta}-\frac{D_{t}(\delta)}{\delta}=V_{I}-w(\delta)
$$

This equation is similar to the equation proposed by Garandet et al. [20] (equation (5)), with an additional term for turbulent diffusion. The estimation of $\delta$ through equation (13) requires a definition of the velocity and turbulent diffusivity profiles in the solute boundary layer. We first consider a general expression of the mean tangential velocity component:

$$
u(z)=f(z) .
$$

As proposed by Garandet et al. [17], mass continuity equation for an incompressible flow can be used to connect tangential and normal convection velocity components:

$$
\frac{\partial u}{\partial x}+\frac{\partial w}{\partial z}=0 .
$$

We suppose that $u$ varies along the interface on a characteristic length $l_{c}$, over which the boundary layer flow occurs. We also consider that $w$ varies in the vertical direction on a characteristic length given by the distance $z$ along the normal to the interface. From an order of magnitude point of view, equation (15) leads to:

$$
\frac{w}{z} \sim-\frac{u}{l_{c}}
$$

The mean convection velocity normal to the interface can thus be expressed by:

$$
w(z) \approx-\frac{z}{l_{c}} f(z) .
$$

The solute boundary layer thickness can be linked to diffusion and turbulent convective transport by the following general equation:

$$
\frac{D}{\delta}-\frac{\nu_{t}(\delta)}{S c_{t} \delta}=V_{I}+\frac{\delta}{l_{c}} f(\delta)
$$


The resolution of equation (18) relies on a meaningful choice for velocity and turbulent viscosity profiles in the solute boundary layer in order to define $f(z)$ and $\nu_{t} / S c_{t}$ at $z=\delta$. Numerical results $[17,19]$ show that for fully turbulent regimes the solute boundary layer becomes thicker than the viscous sublayer but does not extend much in the buffer layer which lies between $z^{+}=10$ to 50 (with $z^{+}=z u_{\tau} / \nu$ ). In this case, Rannie's formula [21] gives a good description of the mean velocity profile until $z^{+} \approx 27.5$ :

$$
u^{+}(z)=A \tanh \left(\frac{z^{+}}{A}\right)
$$

where $u^{+}=u / u_{\tau}$ and $A=14.53$ is an empirical constant. Other famous velocity profiles, such as Von Karman formula [21], could be used depending on the flow configuration. Here we choose Rannie's formula because it provides a simple, unique and differentiable expression of the velocity when $z^{+}$is in the interval [0; 27.5]. The eddy viscosity can, for instance, be expressed by a mixing length model:

$$
\nu_{t}(z)=l^{2}\left|\frac{\partial u}{\partial z}\right|
$$

where the mixing length $l$ can be defined by Van Driest formula [27], which remains valid inside the viscous sublayer:

$$
l=\kappa z\left(1-e^{-z^{+} / c}\right),
$$

where $\kappa=0.4$ is the Von Karman constant and $c=26$ is another empirical constant. As for the normal velocity component, the order of magnitude of $\partial u / \partial z$ is given by:

$$
\frac{\partial u}{\partial z} \sim \frac{u}{z} \sim \frac{f(z)}{z} .
$$

Here again, other turbulence models could be used as well. The benefit of the mixing length model is to remain simple enough to allow an analytical approach. Introducing Rannie's velocity profile and the mixing length model in equation (18), we obtain the following equation for the solute boundary layer thickness:

$$
\frac{D}{\delta}-\frac{\kappa^{2}}{S c_{t}}\left(1-e^{-\delta u_{\tau} /(\nu c)}\right)^{2} A u_{\tau} \tanh \left(\frac{\delta u_{\tau}}{A \nu}\right)=V_{I}+\frac{\delta}{l_{c}} A u_{\tau} \tanh \left(\frac{\delta u_{\tau}}{A \nu}\right) .
$$

Numerical resolution of equation (23) provides an estimate of $\delta$ for any convective regime, as long as the assumption of a boundary layer flow at the solid/liquid interface remains valid.

In order to extract the influence of the different parameters, equation (23) can be simplified by linearisation of convection and turbulent diffusion terms. For convective regimes, $\delta$ is expected to remain small, allowing the following approximations:

$$
A u_{\tau} \tanh \left(\frac{\delta u_{\tau}}{A \nu}\right) \approx \frac{u_{\tau}^{2}}{\nu} \delta
$$

and

$$
\left(1-e^{-\delta u_{\tau} /(\nu c)}\right)^{2} A u_{\tau} \tanh \left(\frac{\delta u_{\tau}}{A \nu}\right) \approx \frac{u_{\tau}^{2}}{\nu}\left(\frac{u_{\tau}}{\nu c}\right)^{2} \delta^{3} .
$$

After substitution in equation (23) and introducing $\Delta$ and $B$, we obtain:

$$
1-\Delta-B \Delta^{3}-\left(\frac{\kappa}{c}\right)^{2} \frac{S c}{S c_{t}} \operatorname{Re}_{V_{I}}^{2} B^{2} \Delta^{4}=0
$$

Once again, this equation is equivalent to the equation proposed by Garandet et al. [17] (equation (6)) with an additional term for turbulent transport. Here we focus on strong convective regimes. The first order term $\Delta$ can be neglected since the interface velocity $V_{I}$ is small compared to the convection velocity $w$ (see equation (13)). Considering high values of $B$ and defining a new parameter $\psi=(\kappa / c) \sqrt{S c / S c_{t}} R e_{V_{I}}$, the asymptotic regime is given by:

$$
\Delta=(\psi B)^{-1 / 2} .
$$

Introducing expression (27) in equation (6), it is possible to define a critical value $B_{c}$ beyond which turbulent transport needs to be taken into account:

$$
B_{c}=\frac{1}{\psi}\left(1+\frac{1}{\psi}\right)^{2} .
$$


This critical value $B_{c}$ depends on interface velocity and solute molecular diffusivity. The evolution of $B_{c}$ as a function of $\psi$ is given in figure 3. Two asymptotic regimes can be identified: $B_{c} \approx \psi^{-1}$ for $\psi>>1$ and $B_{c} \approx \psi^{-3}$ for $\psi<<1$. $B_{c}$ also defines the validity range of equation (6). For moderate convective regimes $\left(B<B_{c}\right)$, the model proposed by Garandet et al. [17] (solution of equation (6)) provides a good estimate of $\Delta$. For stronger convective regimes with fully turbulent flows $\left(B>B_{c}\right), \Delta$ can be obtained from equation (23) or estimated through the asymptotic regime (27).

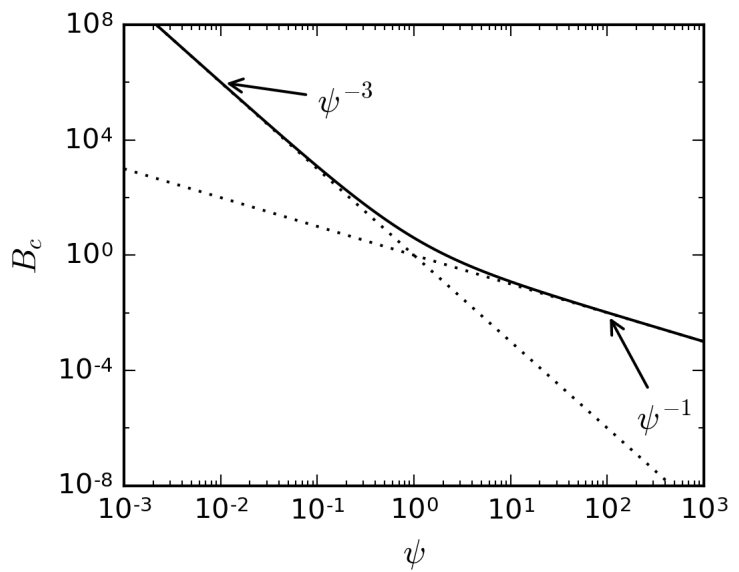

Figure 3: Critical value $B_{c}$ as a function of $\psi$.

\subsection{Validation against numerical results}

In this section the analytical model is confronted with numerical results of solute segregation in a silicon directional solidification process. A 2D lid driven cavity configuration is used as a reference test case (figure 4). A detailed presentation of the problem is provided in a previous publication [19]. Main information about the numerical procedure is briefly reminded here.

Momentum and solute transport equations are solved in a 2D domain. The configuration is described in figure 4. The cavity width is $L=0.2 \mathrm{~m}$ and the liquid height at initialization is $H\left(t_{0}\right)=0.18 \mathrm{~m}$. A lid driven cavity flow is used to impose convection in the liquid. The convection regime is defined by a single dimensionless parameter, namely the Reynolds number $R e_{L}=V_{L} L / \nu$. The lid velocity $V_{L}$ is imposed at the top surface of the cavity and noslip conditions are used on the lateral walls and the solid/liquid interface. The realizable $k-\varepsilon$ turbulence model [28] is used for turbulent configurations $\left(R e_{L} \geq 10^{4}\right)$ and the turbulent Schmidt number is fixed at 0.7. Transient simulations are performed in order to compute solute incorporation in the solid. The solidification front is assumed to be plane and moves at an imposed velocity $V_{I}$. Mass and solute fluxes through the solid/liquid interface are accounted for by local source terms, as defined in previous works [17, 29,30]. Solute incorporation in the solid is defined by the thermodynamic segregation coefficient $k_{0}$, which is set at 0.3 in this study. Regarding spatial discretization, the mesh is refined at the interface to ensure a precise description of the solute boundary layer. Far from the interface the mesh is uniform with $2 \mathrm{~mm} \times 2 \mathrm{~mm}$ cells and the first cell adjacent to the interface is $5 \mu \mathrm{m}$ high, except for $R e_{L}=10^{7}$ configurations where it is reduced to $1 \mu \mathrm{m}$. A mesh independence test was performed and described in the previous publication [19]. This test confirmed that the spatial discretization is sufficient for the resolution of the fluid flow and solute transport. The problem is solved with the commercial code ANSYS FLUENT ${ }^{(\mathrm{TM})}$ [28]. 


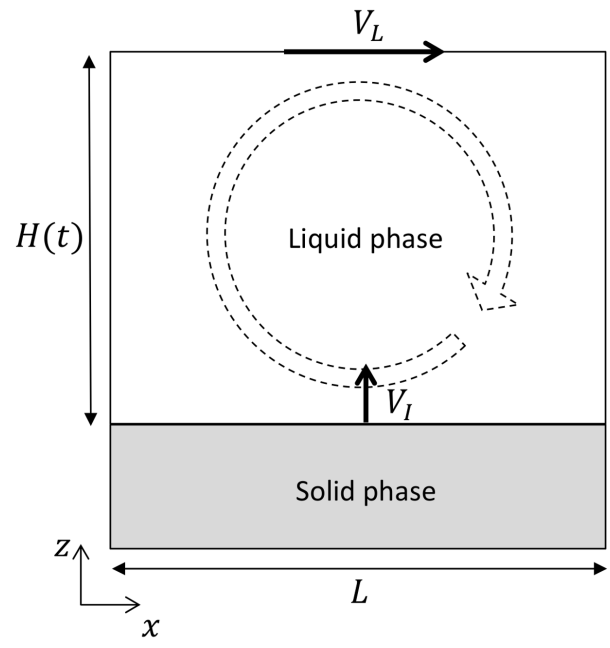

Figure 4: 2D lid driven cavity configuration (geometry, interface velocity $V_{I}$, lid velocity $V_{L}$ and schematic flow pattern).

A parametric study is performed with $R e_{L}$ varying from $10^{2}$ to $10^{7}, D$ varying from $10^{-9} \mathrm{~m}^{2} / \mathrm{s}$ to $10^{-7} \mathrm{~m}^{2} / \mathrm{s}$ and $V_{I}$ varying from $2.8 \mu \mathrm{m} / \mathrm{s}$ to $70 \mu \mathrm{m} / \mathrm{s}$. The same range of parameters was used by Garandet et al. [17] in order to investigate the segregation of various species during photovoltaic silicon crystallization or purification by directional solidification. Liquid silicon properties are used for fluid density $\rho$ and dynamic viscosity $\mu$. The initial solute mass fraction in the liquid is set at $C_{0}=20 \mathrm{ppmw}$ (part per million weight) and the simulations are performed until solid fraction $f_{S}$ reaches $89 \%$. Typical solute mass fraction fields in the solidified ingots obtained for the different convective regimes are presented in section 3 .

Three velocity fields obtained at different steps of the solidification process (corresponding to different solid fractions) for the turbulent regime $R e_{L}=10^{5}$ are reported in figure 5. The flow comprises a main vortex filling the major part of the cavity and secondary vortices in the corners. Characteristic points are identified at the growth front where the shear-stress is null. Trajectories of these points during the solidification are drawn as black dots. As the liquid height is reduced, the secondary vortices are more and more confined in the corners and the characteristic points are shifted to the lateral walls. The precedent publication [19] already pointed out the correlation between segregation patterns and characteristic points locations at the solid/liquid interface. Figure 6 presents several profiles of the convecto-diffusive parameter recorded at various solidification times, for $R e_{L}=10^{5}$ and $R e_{L}=10^{7}$ (in these cases $D=10^{-8} \mathrm{~m}^{2} / \mathrm{s}$ and $V_{I}=10 \mu \mathrm{m} / \mathrm{s}$ ). Characteristic points locations are also drawn as black dots. We can see that $\Delta$ peaks are localized near some of the characteristic points or close to the lateral walls, which confirms the correlation between the flow topology and the mass transfer at the solidification front.

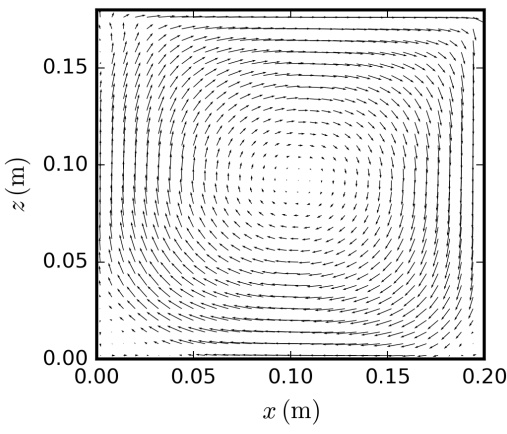

(a) $f_{S}=0$

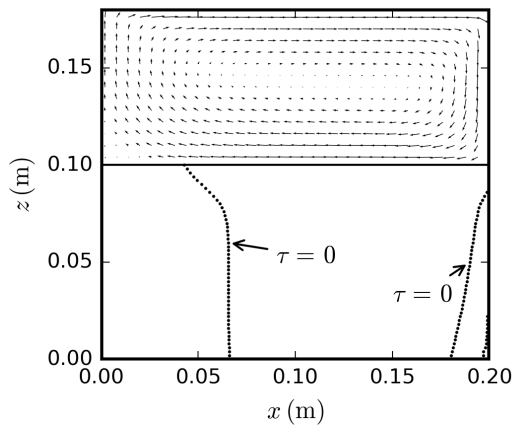

(b) $f_{S}=0.56$

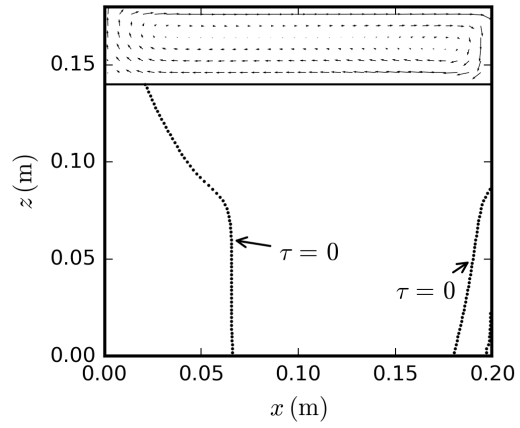

(c) $f_{S}=0.78$

Figure 5: Velocity field for the turbulent regime $R e_{L}=10^{5}$ at different steps of the solidification process. Black dots represent trajectories of characteristic points with zero shear-stress at the solid/liquid interface. 


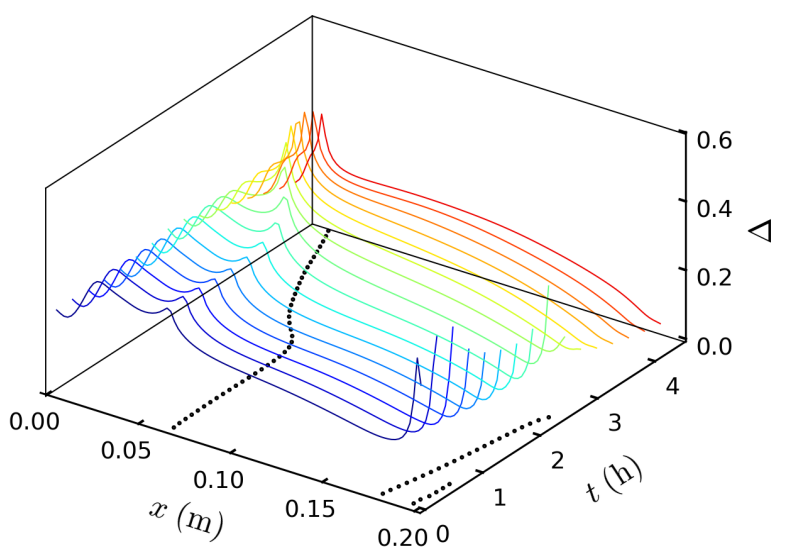

(a) $R e_{L}=10^{5}$

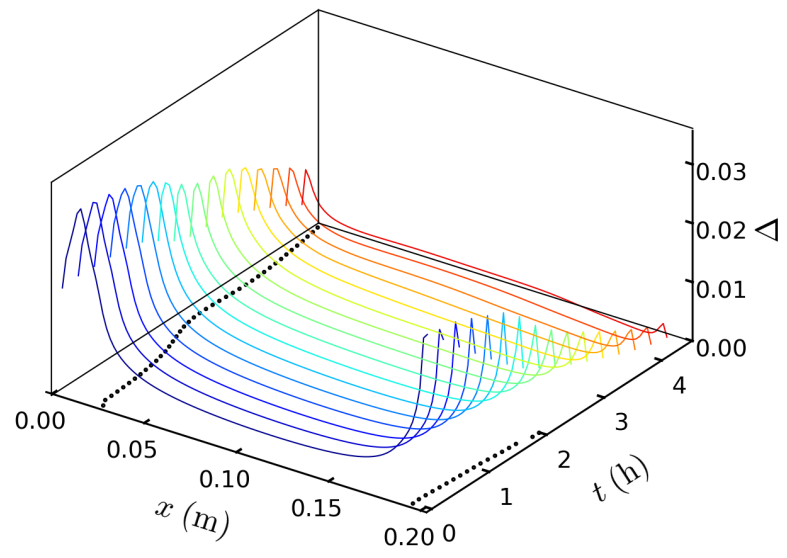

(b) $R e_{L}=10^{7}$

Figure 6: Convecto-diffusive parameter profiles at different solidification times for $\operatorname{Re}_{L}=10^{5}$ (a) and $\operatorname{Re}_{L}=10^{7}$ (b) $\left(D=10^{-8} \mathrm{~m}^{2} / \mathrm{s}\right.$ and $\left.V_{I}=10 \mu \mathrm{m} / \mathrm{s}\right)$. Black dots represent locations of characteristic points with zero shear-stress at the solid/liquid interface.

Span averaged values of $B$ (from equation (7)) and $\Delta$ (from equation (4)) along the solidification front are recorded every time the interface moves $1 \mathrm{~cm}$ upwards, and noted $\langle B\rangle$ and $\langle\Delta\rangle$. To compute the parameter $B$, we assume that the characteristic length $l_{c}$ is directly given by the cavity width $L$. As explained by Garandet et al. [17], this amounts to consider that a unique recirculation is filling the whole cavity and neglects secondary vortices. This assumption is acceptable here since we are studying laterally averaged values. We will see, however, in the next section, that specific characteristic lengths can be defined for the different vortices, in order to enhance lateral segregations description. Results are presented in figure 7 and referred to as "transient" data. In figure 7(a) the molecular diffusivity is set at $D=10^{-8} \mathrm{~m}^{2} / \mathrm{s}$ and different interface velocities are investigated. In figure 7 (b) the interface velocity is set at $V_{I}=10 \mu \mathrm{m} / \mathrm{s}$ and different diffusivities are considered. The different dot groups refer to the different Reynolds numbers used for each value of $D$ and $V_{I}$. These results are compared to the analytical model proposed by Garandet et al. [17], referred to as $\Delta_{t h}$. Numerical results obtained in a quasi-steady configuration [17] are also presented and referred to as "quasi-steady" data.

For all tested values of $D$ and $V_{I}$, the same general trend is observed: numerical results (transient and quasisteady) diverge from $\Delta_{t h}$ for high convective regimes, or more precisely for high values of the product $\psi B$. The divergence occurs around the critical value $B_{c}$ predicted by relation (28). This critical value is reported in figure 7(a) for $V_{I}=2.8$ and $70 \mu \mathrm{m} / \mathrm{s}$ cases (with $D=10^{-8} \mathrm{~m}^{2} / \mathrm{s}$ ) and is equal to 207 and 0.3 , respectively.

For each investigated values of $D$ and $V_{I}$, equation (23) is solved numerically and referred to as "analytical" data in figure 7. A good agreement is observed with numerical results for any convective regime. The asymptotic regime provided by equation (27) is also presented in figure 7 (a) for $V_{I}=2.8$ and $70 \mu \mathrm{m} / \mathrm{s}$, and referred to as "asymptote" data. It provides a good estimate of $\Delta$ for high convective regimes $\left(B>B_{c}\right)$. It appears that a simple way to define the solute boundary layer for any convective regime is to combine the analytical model proposed by Garandet et al. [17] (solution of equation (6)) for diffusive and moderate convective regimes, with the asymptotic regime given by equation (27) for fully developed turbulent regimes. The transition between these solutions is defined by the critical value $B_{c}(28)$.

The last dots groups of each series of transient numerical results (i.e. the groups obtained for the highest values of $B$ ) refer to $R e_{L}=10^{7}$ configurations. For this hydrodynamic regime, a large variation of $\langle\Delta\rangle$ is observed during the first half of the solidification, whereas $\langle B\rangle$ remains almost unchanged. This dispersion is explained by the progressive confinement of the secondary recirculations at the lateral walls. The concentration peaks associated with characteristic points have, indeed, smaller amplitudes as solidification proceeds (see figure 6(b)) and therefore less and less effect on the span averaged value $\langle\Delta\rangle$. To exclude this effect from results analysis, local values of $B$ and $\Delta$ at the center of the interface $(x=0.1 \mathrm{~m})$ are recorded after an interface displacement of $1 \mathrm{~cm}$. In this case, the chosen point is sufficiently far from the characteristic points, and concentration peaks have no influence on the measured values. These values are reported in figure 7 as black crosses. The agreement with the developed analytical model appears to be excellent when 2D effects are ignored.

These results confirm that the introduction of a turbulent diffusion term in the scaling analysis allows the estimation of the segregation even for fully turbulent flows. This analytical model provides an opportunity to 
compute concentration fields in solidified ingots without performing a numerical resolution of the solute boundary layer. This is the topic of the next section.
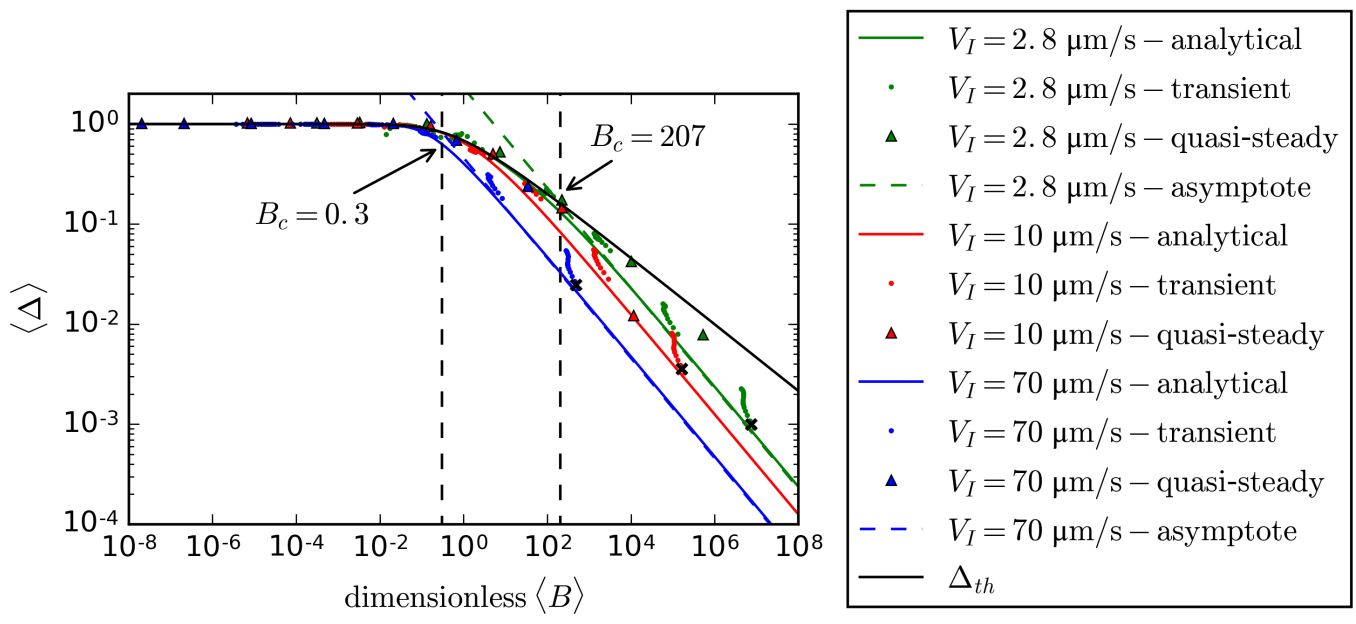

(a) $D=10^{-8} \mathrm{~m}^{2} / \mathrm{s}$

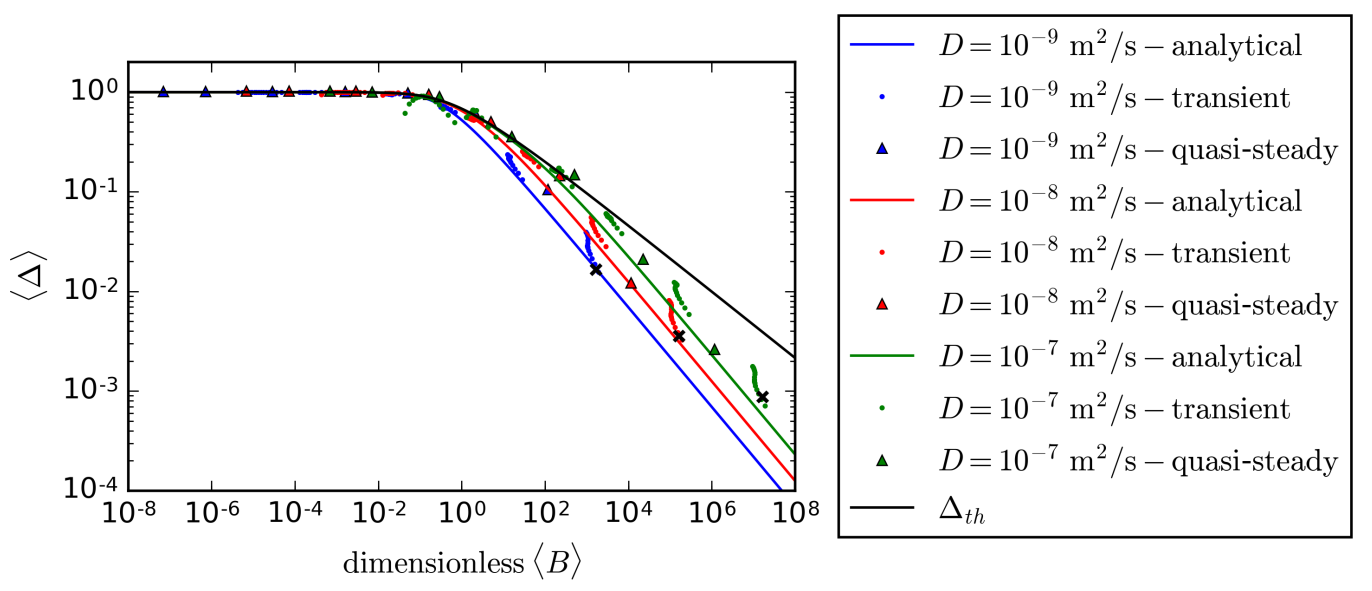

(b) $V_{I}=10 \mu \mathrm{m} / \mathrm{s}$

Figure 7: $\langle\Delta\rangle$ as a function of $\langle B\rangle$ for different solidification conditions (mean values obtained along the interface). Influence of $V_{I}$ (a) and $D(\mathrm{~b})$ on numerical and analytical results. Quasi-steady data refer to Garandet et al. [17] numerical results, transient data refer to our simulations, analytical data refer to the solution of equation (23), asymptote data refer to the asymptotic regime provided by equation (27) and $\Delta_{t h}$ refers to the analytical model proposed by Garandet et al. [17]. Black crosses $(\times)$ represent transient numerical results obtained for $R e_{L}=10^{7}$ at the center of the interface and for a solidified height of $1 \mathrm{~cm}$. Critical values $B_{c}$ obtained from equation (28) for $V_{I}=2.8$ and $70 \mu \mathrm{m} / \mathrm{s}\left(D=10^{-8} \mathrm{~m}^{2} / \mathrm{s}\right)$ are indicated by vertical dashed lines.

\section{Segregation patterns from hydrodynamic simulations}

In this section, a procedure to compute concentration fields in solidified ingots, from convection simulation data in the liquid phase and the developed analytical model, is proposed. The method is applied to the 2D lid driven cavity configuration. Wall shear-stress profiles at the solidification front are obtained during the hydrodynamic simulation of the solidification process. They are used as input data for the analytical segregation computation, performed under Matlab ${ }^{(\mathrm{TM})}$. The description of lateral segregations is improved by the fluid flow structure analysis. The analytical results are compared to the numerical concentration fields obtained by complete simulations including solute transport and segregation.

\subsection{Computation procedure}

A flow chart of the proposed computation procedure is presented in figure 8. This procedure consists of three main steps. First, a directional solidification simulation is performed. The aim of this simulation is to compute the 
convection flow in the liquid phase. Solute transport is not considered at this stage. Shear-stress profiles at the interface $(\tau(x))$ are recorded at each time-step of the transient simulation.

In a second step, the solute boundary layer analytical model is used to determine segregation parameters along the solidification front. Profiles of parameter $B$ are computed from the local values of $\tau$ using equation (7). $\Delta$ profiles are then deduced from the analytical model (see section 2) and effective segregation coefficient profiles $\left(k_{e f f}(x)\right)$ are obtained from equation (3). For a good description of lateral segregations, specific points should be considered at this stage, like the definition of the characteristic length $l_{c}$ and the validity of the analytical model at separation and impingement points. These issues are discussed in the next section (3.2).

Finally, the third step is dedicated to the computation of the solute repartition in the solid phase from an iterative procedure performed under Matlab ${ }^{(\mathrm{TM})} . k_{\text {eff }}$ is defined as the ratio between the solute concentration in the solid phase at the interface $C_{S}^{I}$ and the liquid phase concentration far from the interface $C_{L}^{\infty}$. Thus, the estimation of $C_{L}^{\infty}$, through an iterative computation based on solute mass conservation in the liquid and solid phases, will allow to get $C_{S}^{I}(x)$ and then to obtain the concentration field in the solid.

To compute the concentration field in the solid, the initial transient related to the formation of the solute boundary layer has to be considered. A simplified solution of this initial transient was proposed by Garandet et al. [31] for convecto-diffusive regimes. This solution is derived from a scaling analysis in a 1D configuration and leads to the following definition for the characteristic length of the initial transient $z_{T}(\mathrm{~m})$ :

$$
z_{T}=\frac{D \Delta^{2}}{V_{I}\left[1-\left(1-k_{0}\right) \Delta\right]}
$$

The concentration in the solid is then given by the following relation:

$$
C_{S}\left(x, z_{S}\right)=C_{L}^{\infty}(t) k_{e f f}(x, t)\left[1-\left(1-k_{0}\right) \Delta(x, t) \exp \left(-\frac{z_{S}}{z_{T}(x, t)}\right)\right]
$$

where $z_{S}=t V_{I}$ represents the solidified length $(\mathrm{m})$. Compared to the original expression proposed by Garandet et al. [31], the initial concentration $C_{0}$ is replaced by $C_{L}^{\infty}(t)$ in equation (30) to account for solute accumulation in the liquid. The original publication was dedicated to the initial transient, when the liquid concentration can reasonably be approximated by the initial concentration $C_{0}$. The modified equation (30) is valid for the complete solidification process, but needs an estimation of $C_{L}^{\infty}$, which will be obtained in two steps.

First, assuming an homogeneous concentration $C_{0}$ in the liquid at the beginning of the solidification, the mean solute concentration in the liquid $C_{L}$ mean is easily obtained at each time-step by solute mass conservation. Then, the expression of the concentration profile in the liquid phase $C_{L}(z)$, proposed by Garandet [32] for a convecto-diffusive regime, will be used for each abscissa $x$ :

$$
C_{L}(x, z)=C_{L}^{\infty}\left[1+\frac{\Delta(x)\left(1-k_{0}\right)}{1-\Delta(x)\left(1-k_{0}\right)} \exp \left(-\frac{z}{\delta(x)}\right)\right] .
$$

This equation is derived in a steady-state regime and neglects the initial transient of the solute boundary layer formation. Another expression was proposed later to account for the initial transient [31]. But this expression is too much complex for an analytical integration and the precision improvement would remain limited, especially for high convective regimes where the initial transient is very short. The concentration profile (31) can be integrated on the liquid height $H$ and the cavity width $L$. This integral can be evaluated as $H L C_{L}$ mean and the bulk concentration $C_{L}^{\infty}$ can be obtained from $C_{L \text { mean }}, H, \delta$ and $\Delta$ by:

$$
C_{L}^{\infty}=\frac{C_{L \text { mean }}}{\left\langle 1+\frac{\delta(x)}{H}\left[\frac{\Delta(x)\left(1-k_{0}\right)}{1-\Delta(x)\left(1-k_{0}\right)}\left(1-\exp \left(-\frac{H}{\delta(x)}\right)\right)\right]\right\rangle} .
$$

The notation $\langle\cdot\rangle$ refers to a span average on the cavity width. The assumption of an homogeneous liquid phase outside the solute boundary layer seems reasonable when turbulent convection is involved. Considering the numerical results presented in a previous publication [19], this hypothesis becomes more than questionable for laminar flows, and the definition of a bulk concentration is not so plain. 
Step 1: Transient solidification simulation (ANSYS FLUENT ${ }^{(\mathrm{TM})}$ )

$\rightarrow$ Shear-stress profiles $\tau(x, t)$ at the solid/liquid interface

$\Downarrow$

$$
\begin{aligned}
& \text { Step 2: Segregation parameters from the analytical model } \\
& \quad \rightarrow B(x, t) \text { from equation }(33) \text {, using } \tau(x, t) \text { and } l_{c}(x, t)^{*} \\
& \quad \rightarrow \Delta(x, t) \text { from equation }(6) \text { or }(27) \\
& \quad \rightarrow \text { Spline interpolation for impingement points* } \\
& \quad \rightarrow k_{\text {eff }}(x, t) \text { from equation }(3) \\
& \quad\left({ }^{*} \text { specific treatments for lateral segregations, Matlab }{ }^{(\mathrm{TM})}\right)
\end{aligned}
$$

$\Downarrow$

Step 3: Iterative procedure based on solute mass conservation Matlab $^{(\mathrm{TM})}$ )

$\rightarrow$ Liquid bulk concentration $C_{L}^{\infty}$ from equation (32)

$\rightarrow$ Initial transient characteristic length $z_{T}$ from equation (29)

$\rightarrow$ Solid concentration $C_{S}\left(x, z_{S}\right)$ from equation (30)

Figure 8: Flow chart of the procedure for segregation computation using the solute boundary layer analytical model.

\subsection{Lateral segregations description}

The aim of the procedure is to give a realistic picture of axial and lateral segregation patterns in the solid. Therefore, local variations of the solute boundary layer thickness along the solidification front must be estimated. For this purpose, local values of $\Delta$ are computed from local values of wall shear-stress along the interface. However, the scaling analysis presented in section 2 requires the definition of a characteristic length $l_{c}$ for the lateral variations of the convection velocity along the solid/liquid interface. As explained in section 2.2, Garandet et al. [17] define this length as the cavity width $L$. But the study of the lid driven flow topology shows that multiple recirculations occur in the cavity and have a significant influence on lateral segregations [19]. The positions and sizes of these vortices are controlled by the lid velocity magnitude and the aspect ratio of the cavity $H(t) / L$. An evolution of the flow topology can be observed during the solidification process (see figure 5). To account for this phenomenon, a detection procedure of the characteristic points at the solid/liquid interface is used. The characteristic points are defined as the locations where the wall shear-stress is null (such points are shown in figure 5). The recirculations lengths are then obtained by the distance between two characteristic points, or between a characteristic point and the adjacent lateral wall for corner recirculations. These lengths are determined at each time-step of the solidification and provide a meaningful description of the flow structure in the cavity (figure 9 presents a schematic flow comprising three recirculations with different characteristic lengths). The parameter $B$ is then defined locally at each point of the solidification front as:

$$
B(x, t)=\frac{\tau(x, t) D^{2}}{V_{I}^{3} \mu l_{c}(x, t)}
$$

where $l_{c}(x, t)$ is the characteristic length of the recirculation that sweeps this point of the front.

Besides, the analytical model of the solute boundary layer is based on the assumption of a boundary layer flow parallel to the solid/liquid interface (see equation (19)). This assumption is reasonable on the major part of the interface, but is incorrect in the vicinity of the characteristic points, fluid flow being there fully two-dimensional. This limitation has already been pointed out in previous publications $[3,19]$. To discuss this issue, two cases must be distinguished: separation and impingement points. As an example, figure 10 presents a typical profile of the convecto-diffusive parameter $\Delta$ along the interface. This profile comes from a segregation simulation in the 2D lid driven cavity configuration, for a Reynolds number of $R e_{L}=10^{4}$. In this case, two separation points and two impingement points are found at the interface.

At the separation points the flow is leaving the interface $(w>0$ with our choice of referential). The numerical results highlight concentration peaks at these locations associated with peaks in the solute boundary layer thickness (see figure 10). Solute accumulation at the separation points comes from lateral convective fluxes from the adjacent vortices. The $\Delta$ peak magnitude depends on the global convective regime in the cavity. The analytical model also predicts such a peak since $\Delta$ tends to 1 when $\tau$ tends to zero. But the magnitude of this analytical peak has no physical link with the convection intensity. Therefore, analytical segregation profiles at separation points remain 
solely qualitative.

At impingement points the flow is directed toward the interface $(w<0)$ and the numerical simulations predict a local minimum of the solute concentration associated with a minimum of the solute boundary layer thickness. This seems physically sound since the flow is bringing low concentrated liquid from the bulk at the interface. The wall shear-stress is, however, null at these points, so that the analytical model predicts a maximum for the solute boundary layer thickness ( $\Delta$ tending to 1 ). To overcome this limitation of the analytical model, a spline interpolation procedure is used to remove $\Delta$ peaks associated to impingement points. Figure 10 presents the analytical profile of the convecto-diffusive parameter, which is computed thanks to the solute boundary layer model presented in section 2. Two local maxima related to impingement points can be identified on this profile. The dotted curve represents the profile obtained with the spline interpolation procedure.

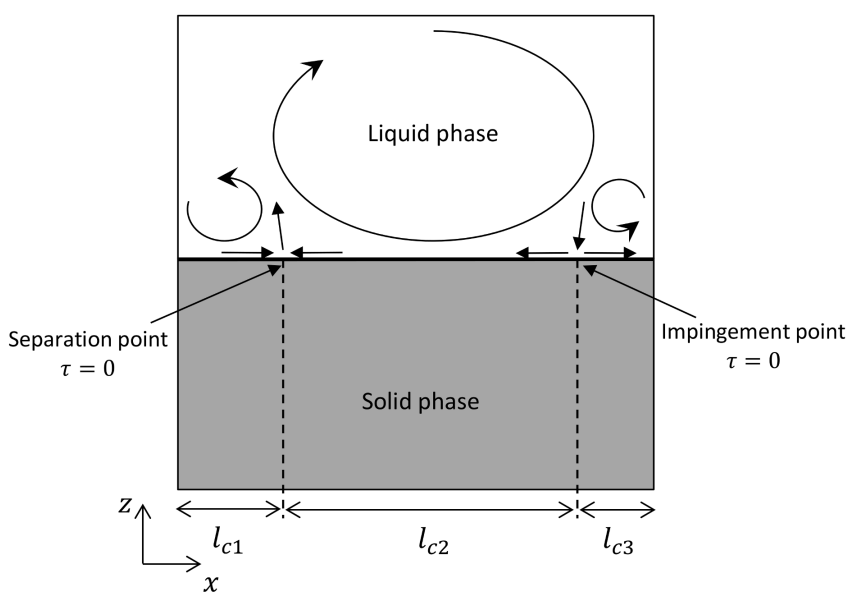

Figure 9: 2D lid driven cavity configuration and schematic flow pattern with three recirculations; definition of recirculation lengths $l_{c 1}$, $l_{c 2}$ and $l_{c 3}$.

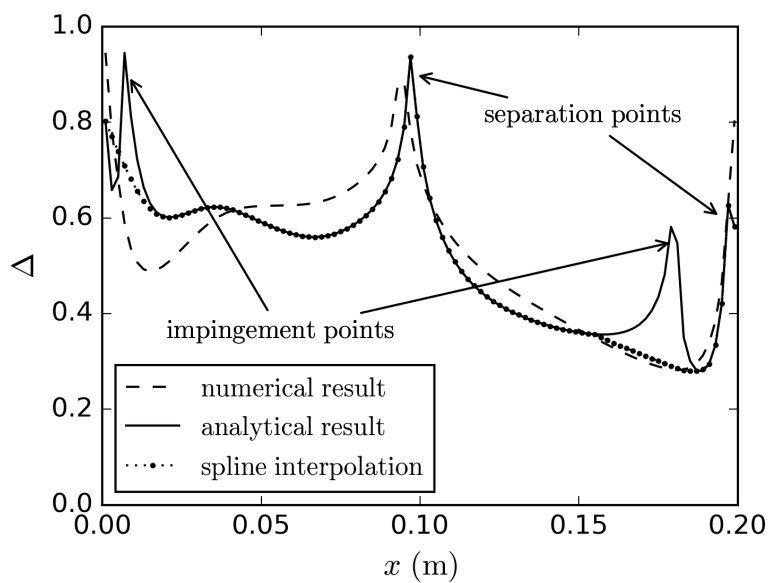

Figure 10: Numerical and analytical convecto-diffusive parameter profiles along the solidification front, $\Delta(x)$, for $R e_{L}=10^{4}$ and a solid fraction $f_{S}=0.44$; illustration of the spline interpolation procedure at impingement points.

\subsection{Analytical concentration fields}

The segregation computation procedure is applied to the transient lid driven cavity configuration. Numerical simulations of segregation in silicon are performed for Reynolds numbers ranging from $R e_{L}=10^{2}$ to $R e_{L}=10^{7}$. The solidification rate is fixed at $V_{I}=10 \mu \mathrm{m} / \mathrm{s}$, the molecular diffusivity is $D=10^{-8} \mathrm{~m}^{2} / \mathrm{s}$, the segregation coefficient is $k_{0}=0.3$ and the initial concentration is set at $C_{0}=20$ ppmw. Liquid silicon properties are used for fluid density and dynamic viscosity. The simulations are performed until solid fraction reaches $89 \%$. The profiles of wall 
shear-stress at the solid/liquid interface are recorded at each time-step of the simulation. These profiles are used as input data for the computation of the solute repartition in the solid from the analytical model. The resulting concentration fields are compared to the numerical results, which have already been presented in a previous publication [19]. Regarding analytical segregation computation, the local values of $B$ are compared to the critical value $B_{c}$ and the local convecto-diffusive parameter $\Delta$ is computed from the solution of equation (6) when $B<B_{c}$ or from equation (27) when $B>B_{c}$. The iterative procedure described in paragraph 3.1 is then applied to progress from one time step to the next one.

Figures 11 and 12 compare numerical and analytical concentration fields in the solidified ingot, obtained for $R e_{L}=10^{7}$ and $10^{6}$. For these turbulent regimes, lateral segregations are weak, with almost horizontal isoconcentration lines. Figures $11(\mathrm{c})$ and $12(\mathrm{c})$ present the relative deviation between the numerical and analytical concentrations. Mean and maximum relative deviations are respectively $0.5 \%$ and $52 \%$ for $R e_{L}=10^{7}$ and $1 \%$ and $110 \%$ for $R e_{L}=10^{6}$. Figure 12(c) shows that the maximum errors are located at the main recirculation separation point, as explained in paragraph 3.2.

Figures 13 and 14 present the results obtained for the turbulent regimes $R e_{L}=10^{5}$ and $10^{4}$. The lower convection intensity leads to more significant lateral segregations. The analytical model provides a very good estimate of local curvatures of iso-concentration lines. The qualitative influence of the main recirculation separation point is also well retrieved. For $R e_{L}=10^{5}$, the mean relative deviation on the concentration field is $3.7 \%$ and the maximum local relative deviation is $140 \%$. For $R e_{L}=10^{4}$, mean and maximum relative deviations are $6.3 \%$ and $72 \%$, respectively. The highest errors are located at the separation points and also near the lateral walls. Indeed, when an impingement point is close to a lateral wall, the spline interpolation procedure is difficult to perform and becomes less accurate.

Figures 15 and 16 present concentration fields in the solid for laminar regimes $R e_{L}=10^{3}$ and $10^{2}$. The agreement between analytical and numerical results is not as good as for turbulent regimes. For $R e_{L}=10^{3}$, the lateral segregations are under estimated. The previous publication [19] explained that the concentration in the liquid phase is very inhomogeneous for this configuration. At the separation point of the main recirculation, the numerical simulation predicts a convecto-diffusive parameter greater than 1 . The analytical model is based on the assumption of an homogeneous liquid phase outside the solute boundary layer. It can not lead to a convecto-diffusive parameter larger than 1, which explains the under estimation of the lateral segregations. This particular regime is, indeed, very close to the convecto-diffusive transition and it is not surprising that the extension of our $1 \mathrm{D}$ model to a $2 \mathrm{D}$ configuration leads to relatively poor results in this case. For $R e_{L}=10^{2}$, the lateral segregations are also under estimated by the analytical model. These lateral segregations are, however, less pronounced than for $\operatorname{Re}_{L}=10^{3}$ and the overall segregation regime is very close to pure diffusion. Mean and maximum relative deviations are respectively $10 \%$ and $71 \%$ for $R e_{L}=10^{3}$ and $1.6 \%$ and $16 \%$ for $R e_{L}=10^{2}$.

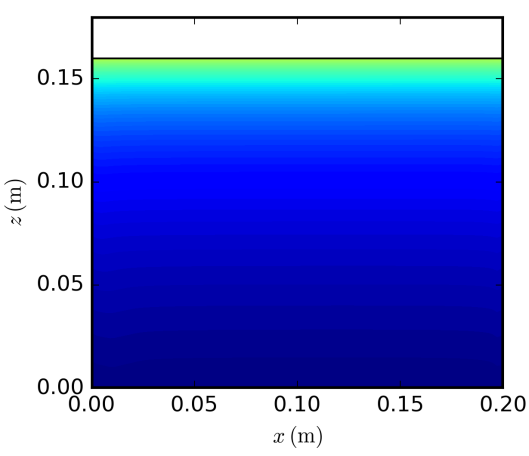

(a) Numerical result

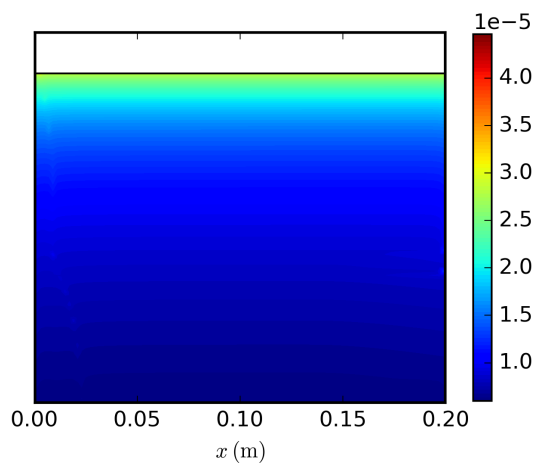

(b) Analytical result

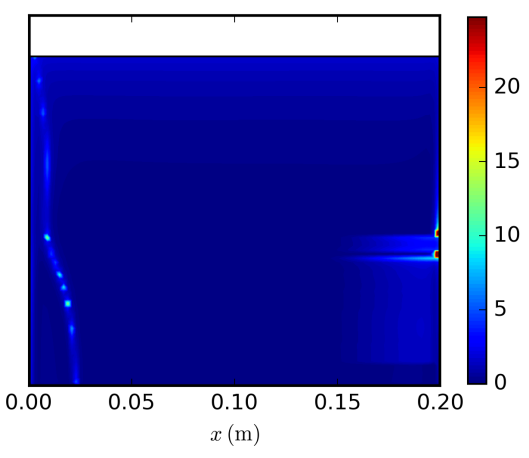

(c) Relative deviation (\%)

Figure 11: Numerical and analytical concentration fields in the ingot solidified under the turbulent convection regime $R e_{L}=10^{7}$. In this figure and in those following (figures 11 to 16), concentrations are given as mass fractions and relative deviation colormaps are limited to $25 \%$ to enhance the contrast. 


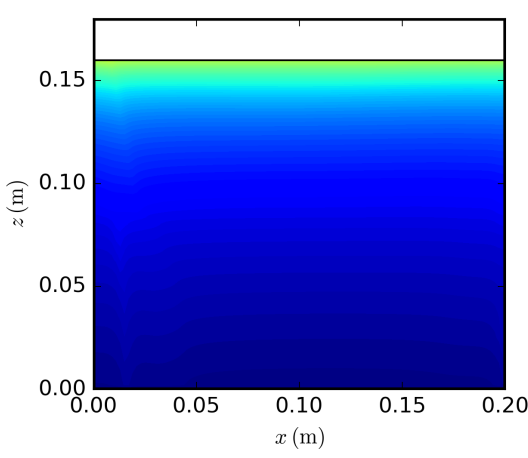

(a) Numerical result

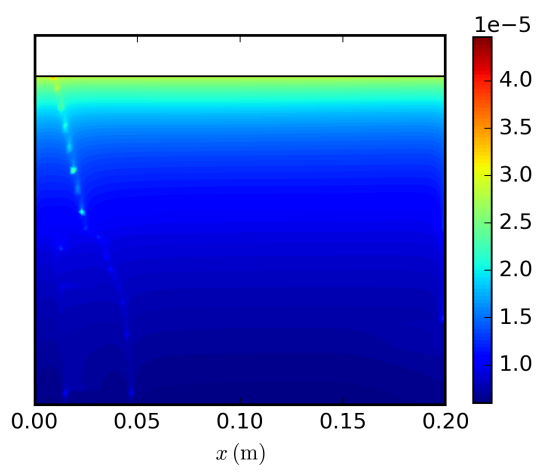

(b) Analytical result

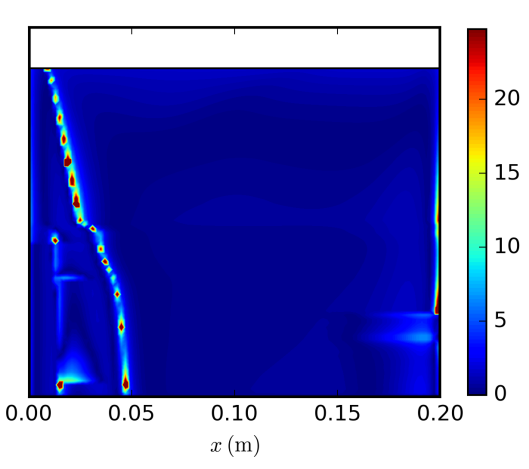

(c) Relative deviation (\%)

Figure 12: Numerical and analytical concentration fields in the ingot solidified under the turbulent convection regime $R e_{L}=10^{6}$.

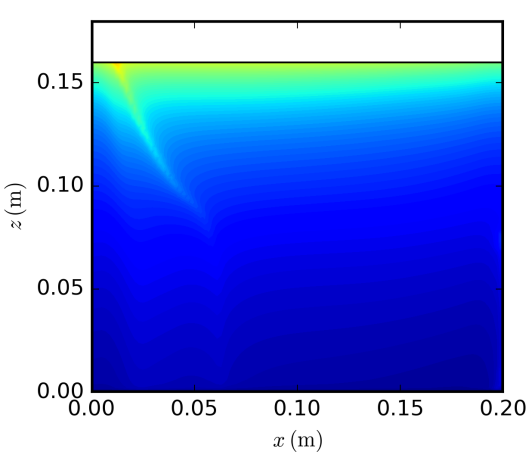

(a) Numerical result

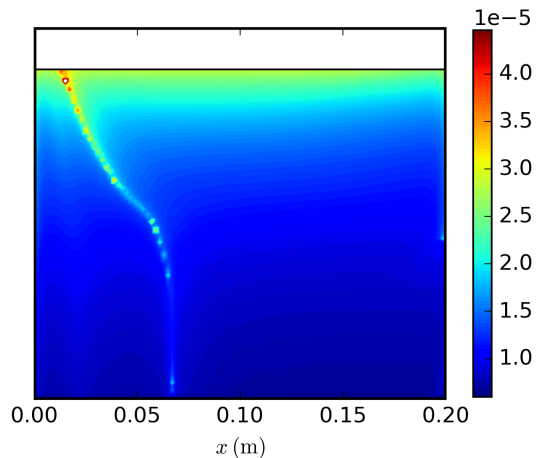

(b) Analytical result

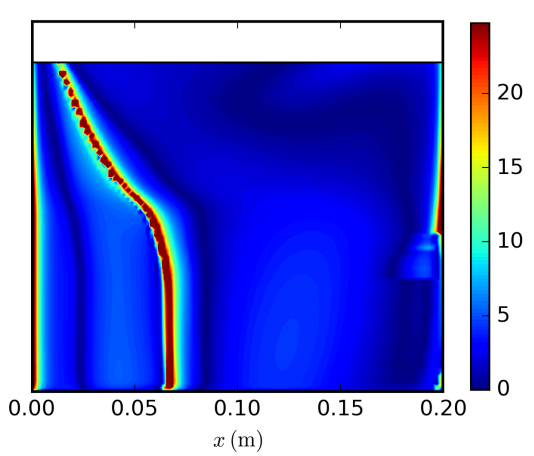

(c) Relative deviation (\%)

Figure 13: Numerical and analytical concentration fields in the ingot solidified under the turbulent convection regime $R e_{L}=10^{5}$.

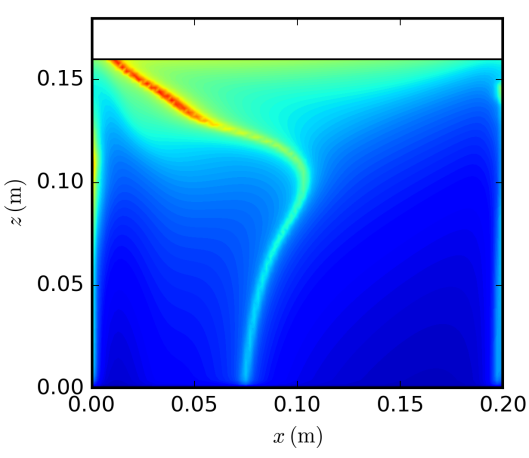

(a) Numerical result

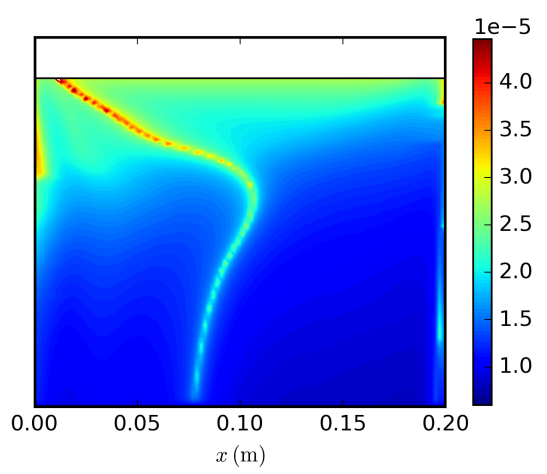

(b) Analytical result

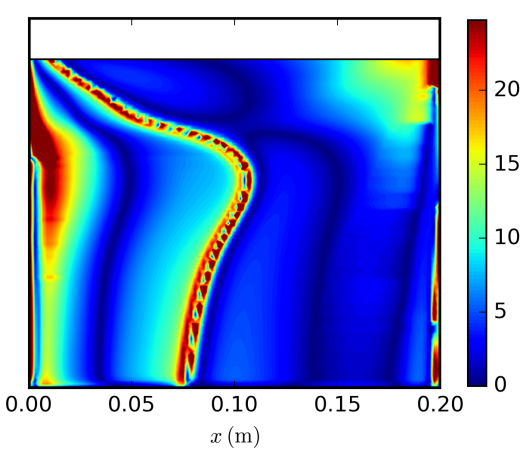

(c) Relative deviation (\%)

Figure 14: Numerical and analytical concentration fields in the ingot solidified under the turbulent convection regime $R e_{L}=10^{4}$. 


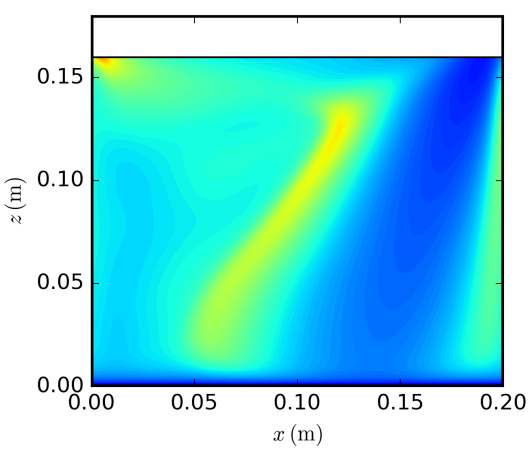

(a) Numerical result

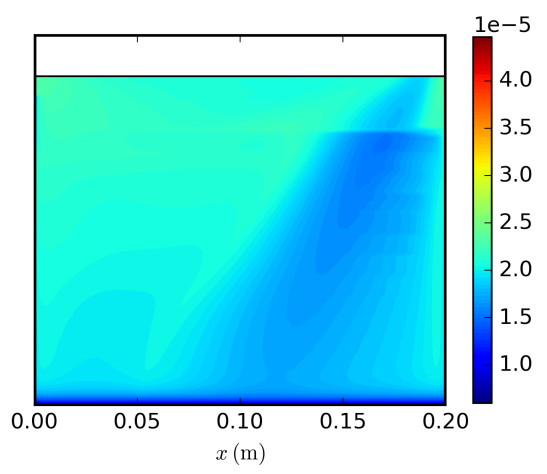

(b) Analytical result

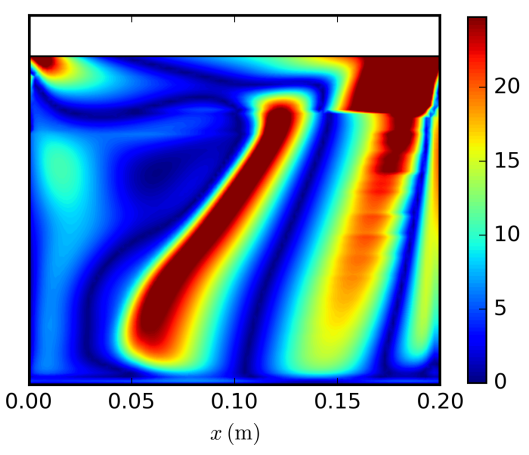

(c) Relative deviation (\%)

Figure 15: Numerical and analytical concentration fields in the ingot solidified under the laminar convection regime $\operatorname{Re}_{L}=10^{3}$.

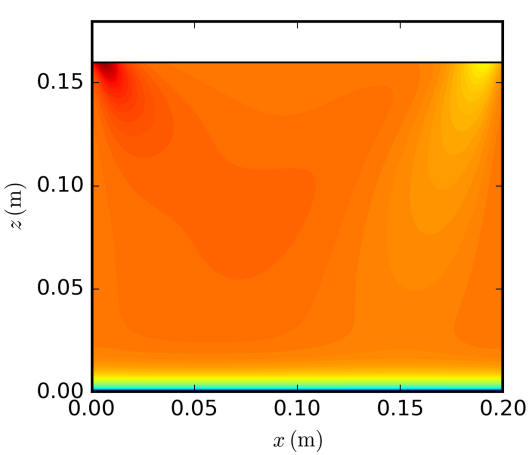

(a) Numerical result

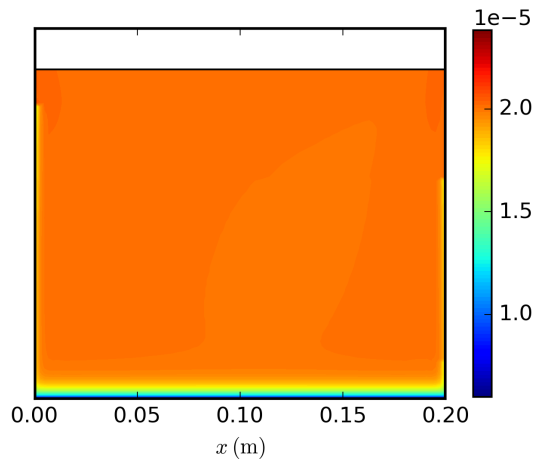

(b) Analytical result

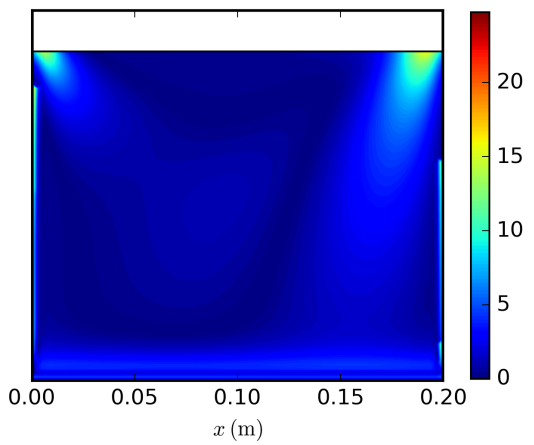

(c) Relative deviation (\%)

Figure 16: Numerical and analytical concentration fields in the ingot solidified under the laminar convection regime $R e_{L}=10^{2}(\mathrm{a}$ different color scale is used to enhance the contrast).

Figure 17 presents normalized longitudinal segregation profiles. The notation $\left\langle C_{S}\right\rangle$ refers to the averaged concentration in the horizontal direction, $x$. Turbulent flows $\left(R e_{L}=10^{4}\right.$ to $\left.10^{7}\right)$ lead to convective segregation regimes with an effective segregation coefficient getting closer to $k_{0}$ as $R e_{L}$ increases. For these convective regimes, the analytical model provides a very good estimate of the longitudinal segregation. The initial transients observed for turbulent regimes are well described by the approximated solution given by equation (30). Laminar flows $\left(R e_{L}=10^{2}\right.$ and $\left.10^{3}\right)$ lead to an almost purely diffusive regime with a constant averaged concentration after the initial transient. For $R e_{L}=10^{2}$, the agreement with numerical results is also very good. The concentration is only slightly overestimated at the end of the initial transient, for solid fractions laying between 0.05 and 0.1. This error can be reduced by using the exact solution proposed by Garandet et al. [31] for the initial transient in a convectodiffusive regime. For $R e_{L}=10^{3}$, the agreement between the analytical and the numerical results is not so good. The analytical concentration is underestimated for solid fractions between 0.05 and 0.6 . This issue is related to the poor description of the solute transport at the separation zone of the main recirculation. A further consequence is that, due to solute mass conservation, the concentration is overestimated at the end of the solidification. 


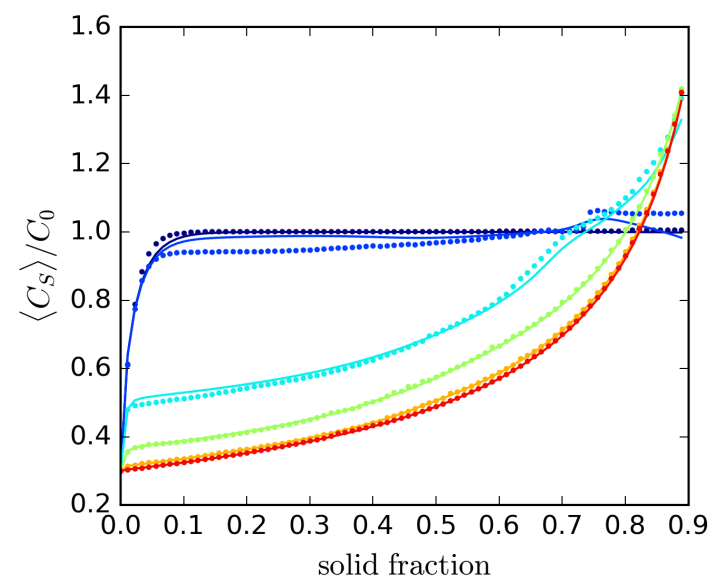

\begin{tabular}{|c|c|c|c|}
\hline & $R e=10^{2}-$ numerical & - & Re $=10^{5}-$ numerical \\
\hline - & $R e=10^{2}-$ analytical & - & $\operatorname{Re}=10^{5}-$ analytical \\
\hline & Re $=10^{3}-$ numerical & 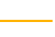 & Re $=10^{6}-$ numerical \\
\hline • & $R e=10^{3}-$ analytical & - & Re $=10^{6}$ - analytical \\
\hline & Re $=10^{4}-$ numerical & - & Re $=10^{7}-$ numerical \\
\hline - & $R e=10^{4}-$ analytical & • & $\operatorname{Re}=10^{7}-$ analytical \\
\hline
\end{tabular}

Figure 17: Longitudinal segregation profiles $\left\langle C_{S}\right\rangle / C_{0}$ : comparison between analytical and numerical results at different Reynolds numbers.

Figure 18 presents the mean relative deviation between analytical and numerical concentration fields as a function of the Reynolds number. For turbulent regimes, the error of the analytical model is reduced when the convection intensity increases. This is mainly due to the reduction of the lateral segregations and the limitation of separation points influence on mass transfer. For laminar regimes, we observe an opposite trend with a mean error increasing with the Reynolds number. In this case, the increase of convection intensity leads to complex solute transport in the liquid and strong lateral segregations. Coupling the presently proposed model with a numerical solution of the solute transport in the fluid bulk would improve the model, but is out of scope of the present paper. The low error level for $R e_{L}=10^{2}$ confirms the weakness of the lateral segregations, in an almost purely diffusive regime.

The different curves plotted in figure 18 represent the results obtained with successive enhancements in the model. For curve 1, the first model proposed by Garandet et al. [17] is used and no attempt is made to enhance lateral segregation description. For curve 2, the characteristic lengths of the different vortices are computed. This modification has a significant influence on the accuracy of the model with a reduced error for all convecto-diffusive regimes. For curve 3, the spline interpolation procedure at impingement points is introduced. This procedure is relevant for local concentration corrections, but has little effect on mean errors, except for $R e_{L}=10^{4}$. This is why we have considered that improving this relatively rough approach by a local approach was not worth for the moment. For curve 4, turbulent transport is accounted for in the solute boundary layer model. This leads to a reduced error for the turbulent regimes $R e_{L}=10^{6}$ and $10^{7}$. Curve 4 corresponds to the concentration fields presented in this study (figures 11 to 16 ). Finally, for curve 5 , the convecto-diffusive parameter $\Delta$ is limited at separation points to account for the global convective regime. On a first attempt, an empirical criterion based on the numerical results is used. $\Delta$ is thus limited to twice its mean value along the interface. This criterion is found to have very little influence on the mean error. Therefore, it is not further investigated in this study.

In order to attest the efficiency of the solute wall functions, new hydrodynamic simulations are achieved without interface refinement. The new mesh is uniform in the complete domain with $2 \mathrm{~mm} \times 2 \mathrm{~mm}$ cells. The height of the first cell adjacent to the interface is 400 times larger than for the refined cases (2000 times for $R e_{L}=10^{7}$ configurations). For high Reynolds number configurations, the viscous sublayer is thinner than the mesh size and a classical log law is used to define the velocity profile at the solid/liquid interface. This is not the case for the simulations with interface refinement since the viscous sublayer is systematically resolved. In fact, the choice of the proper velocity profile to apply is handled by the Enhanced Wall Treatment option proposed by ANSYS FLUENT ${ }^{(\mathrm{TM})}$ [28]. For these new simulations, only the fluid flow is solved numerically and the analytical segregation computation is performed from the resulting wall shear-stress profiles. Analytical concentration fields are compared to numerical results of the complete segregation simulations with interface refinement. Curve 6 reports the mean relative deviations 
between these analytical and numerical concentrations (figure 18). We can see that mean errors keep the same order of magnitude when the analytical method is applied to shear-stress profiles obtained with or without interface refinement. Compare to curve 4 (same analytical model), errors are increased for $R e_{L}=10^{4}, 10^{5}$ and $10^{6}$. This is due to a slight shift of the separation and impingement points at the interface with the new computational grid. This result highlights the benefit of the analytical model: it provides a meaningful estimate of segregations without numerical resolution of the solute boundary layer, allowing larger mesh size at solid/liquid interface and leading to memory and computational time savings. The accuracy of the method will mainly depend on the ability of the numerical model to describe the hydrodynamic boundary layers at the solidification front.

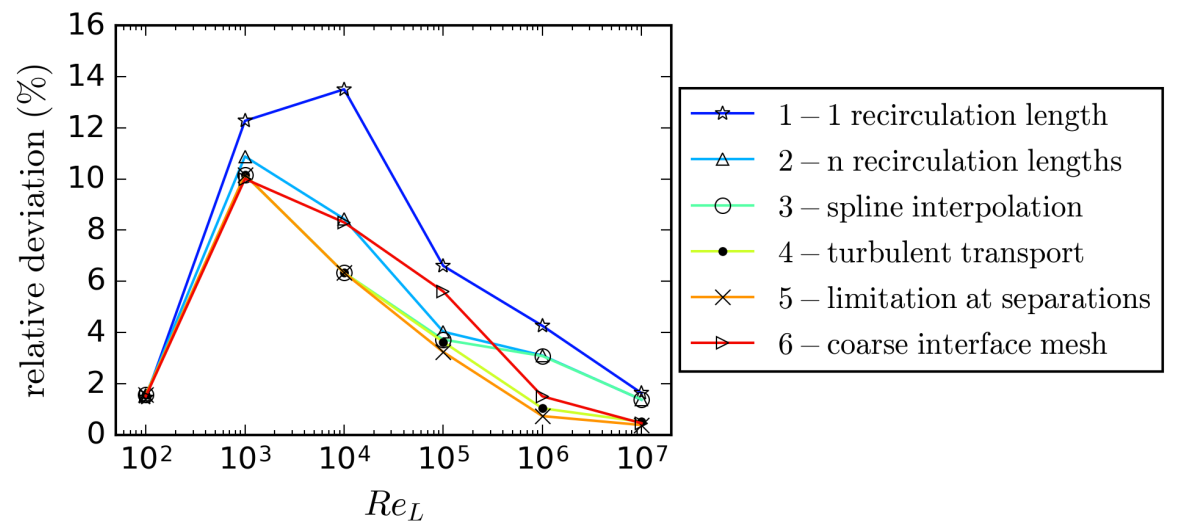

Figure 18: Mean relative deviation (\%) between analytical and numerical 2D concentration fields. Curve 1: results obtained with Garandet et al. [17] analytical model. Curve 2: definition of several characteristic lengths for the different vortices. Curve 3: spline interpolation of $\Delta$ at the impingement points. Curve 4: turbulent transport in the solute boundary layer. Curve 5: limitation of $\Delta$ at the separation points. Curve 6: hydrodynamic simulations without interface refinement.

\section{Correlation for different species}

\subsection{Objective and method}

This section presents an interesting feature of the analytical model for the study of the segregation associated with various species. In the previous section, we relied on wall shear-stress profiles extracted from numerical simulations to predict segregation profiles thanks to the scaling analysis of the solute boundary layer. But the solute wall functions presented in section 2 also provide a direct correlation between the effective segregation coefficients of different species. This type of correlation is of great interest for photovoltaic silicon purification and crystallization by directional solidification, since various species will interfere with solar cells electrical properties. One can think of dopants, like boron or phosphorus, voluntarily incorporated in the melt or contaminant impurities such as carbon, aluminum, iron, copper, etc. Each species has a specific molecular diffusivity $D$ and thermodynamic segregation coefficient $k_{0}$. Therefore, each species has specific segregation parameters, namely $k_{\text {eff }}$ and $\Delta$. Considering a given directional solidification process, we have seen in section 2 that the segregation parameters are controlled by the convection conditions in the melt. Our aim is to show that segregation parameters of various species can be estimated from segregation parameters of one specific species. For experimental studies, this type of correlation can reduce the number of chemical analyses required to characterize segregations. Then, the qualification procedures for the purification and crystallization processes by directional solidification could be simplified. For numerical studies, the segregation problem could be reduced to one solute transport equation of a reference species. This can lead to significant time saving for parametric studies based on advanced solidification simulations methods involving a precise description of the solid/liquid interface.

As mentioned above, we shall assume that the effective segregation coefficient $k_{e f f} 1$ of a reference species 1 has been determined for a given solidification process. The related convecto-diffusive parameter $\Delta_{1}$ is simply deduced from equation (3). Our objective is to provide an estimate of $\Delta_{2}$ and $k_{e f f} 2$ for another species denoted 2. The first step is to determine the parameter $B_{1}$, which governs the segregation of species 1 . In a first attempt, this parameter can be deduced from equation (6). However, this equation is only valid if $B_{1}$ is smaller than the critical value $B_{c 1}$. Otherwise, $B_{1}$ should be deduced from the asymptotic regime given by equation (27). For a given solidification process of a dilute alloy, $\tau, V_{I}, \mu$ and $l_{c}$ are constant parameters for the segregation of species 1 and 2 . Thus, the 
parameter $B_{2}$ related to the segregation regime of species 2 is given by the following relation:

$$
B_{2}=B_{1}\left(\frac{D_{2}}{D_{1}}\right)^{2}
$$

where $D_{1}$ and $D_{2}$ respectively stand for the molecular diffusivities of impurities 1 and 2 . Once again, $B_{2}$ has to be compared to the critical value $B_{c 2}$. Then, the convecto-diffusive parameter $\Delta_{2}$ can be obtained from the analytical solution of equation (6) proposed by Garandet et al. [17] if $B_{2}$ remains smaller than $B_{c 2}$, or from equation (27) if $B_{2}$ is greater than $B_{c 2}$. Finally, $k_{e f f 2}$ is given by equation (3).

\subsection{Application}

Segregation simulations of phosphorus, aluminum and iron are performed in the transient lid driven cavity configuration in order to apply the correlation proposed in the previous paragraph. Thermodynamic segregation coefficients and molecular diffusivities used for $\mathrm{P}, \mathrm{Al}$ and $\mathrm{Fe}$ are given in table 1. For this study the interface velocity is kept at $V_{I}=10 \mu \mathrm{m} / \mathrm{s}$ and the initial concentration is set at $C_{0}=65$ ppmw. A turbulent hydrodynamic regime is used with a Reynolds number $R e_{L}=1.36 \times 10^{5}$.

Table 1: Thermodynamic segregation coefficients $k_{0}$ and molecular diffusivities $D$ for $\mathrm{P}, \mathrm{Al}$ and Fe [33-36].

\begin{tabular}{ccc}
\hline & $k_{0}$ & $D\left(\mathrm{~m}^{2} / \mathrm{s}\right)$ \\
\hline $\mathrm{P}$ & $3.32 \times 10^{-1}$ & $2.3 \times 10^{-8}$ \\
\hline $\mathrm{Al}$ & $2.00 \times 10^{-3}$ & $6.8 \times 10^{-8}$ \\
\hline $\mathrm{Fe}$ & $8.00 \times 10^{-6}$ & $8.0 \times 10^{-8}$ \\
\hline
\end{tabular}

Figure 19 presents the longitudinal segregation profiles for the three considered species. Effective segregation coefficients are extracted from these profiles by interpolation with a modified Scheil equation:

$$
\frac{C_{S}\left(f_{S}\right)}{C_{0}}=k_{e f f}\left(1-f_{S}\right)^{k_{e f f}-1},
$$

$f_{S}$ being the solid fraction. Interpolated coefficients $k_{\text {eff } f i t}$ are given in table 2 . The analytical model is then used to determine the effective segregation coefficients for aluminum and iron from the interpolated $k_{\text {eff } f \text { it }}$ for phosphorus. These estimated effective segregation coefficients are also given in table 2 and referred to as $k_{\text {eff } t h}$. For aluminum and iron the analytical model provides a very good estimate of the effective segregation coefficients with about $1 \%$ of relative deviation from the numerical results.

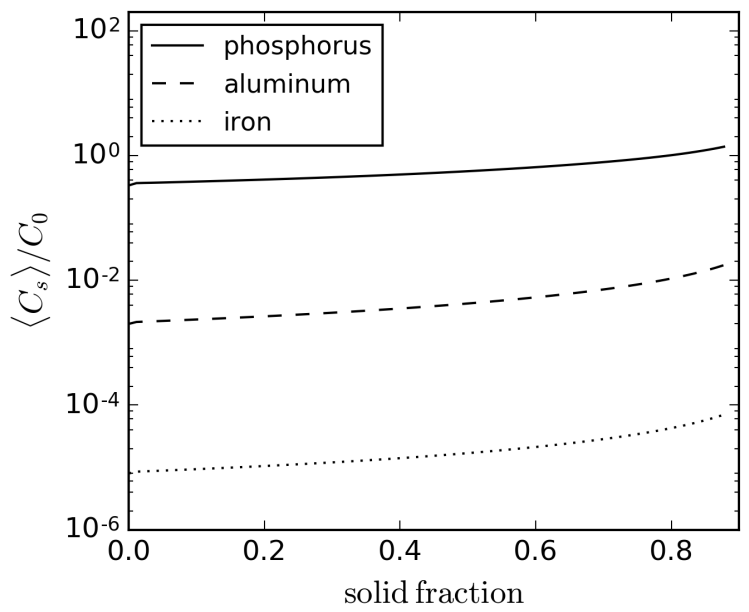

Figure 19: Longitudinal segregation profiles $\left\langle C_{S}\right\rangle / C_{0}$ for phosphorus, aluminum and iron. 
Table 2: Interpolated $\left(k_{e f f f i t}\right)$ and estimated $\left(k_{e f f t h}\right)$ effective segregation coefficients.

\begin{tabular}{cccc}
\hline & $k_{\text {eff fit }}$ & $k_{\text {eff } t h}$ & $\begin{array}{c}\text { relative } \\
\text { deviation }(\%)\end{array}$ \\
\hline $\mathrm{P}$ & $3.5782 \times 10^{-1}$ & - & - \\
\hline $\mathrm{Al}$ & $2.1221 \times 10^{-3}$ & $2.1004 \times 10^{-3}$ & $1.0 \%$ \\
\hline $\mathrm{Fe}$ & $8.4479 \times 10^{-6}$ & $8.3543 \times 10^{-6}$ & $1.1 \%$ \\
\hline
\end{tabular}

This approach can also be used to study lateral segregations. Here, the correlation is applied to local values of $\Delta$ along the solid/liquid interface to predict $\Delta$ profiles for aluminum and iron from numerical results for phosphorus. Figure 20 presents convecto-diffusive parameter profiles at the solid/liquid interface for aluminum and iron. These profiles are arbitrarily taken at a solid fraction $f_{S}=0.44$. The profiles for the two species present similar shapes since they are influenced by the same convective regime. For both species, the numerical segregation results are compared to the predictions obtained from the analytical correlation applied to the phosphorus results. The correlation provides an estimate of $\Delta$ profiles for aluminum and iron with a relative deviation below $20 \%$ from the numerical results.

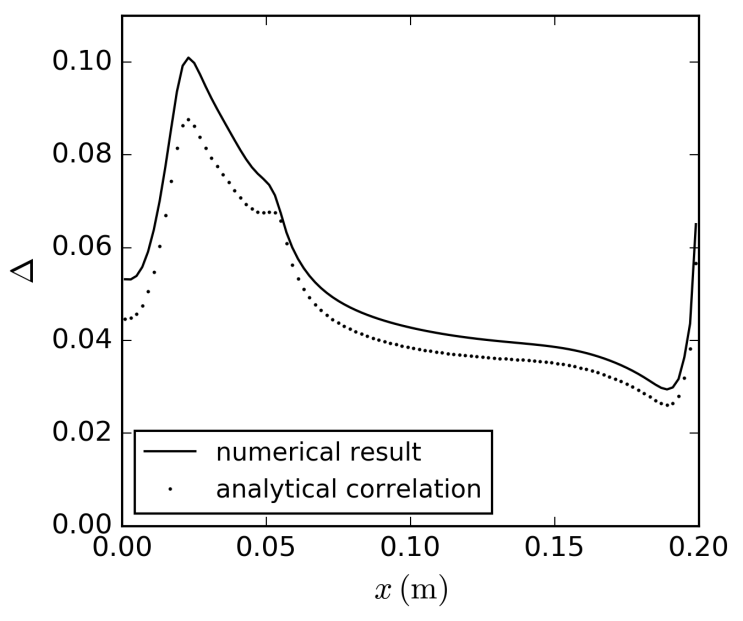

(a) aluminum

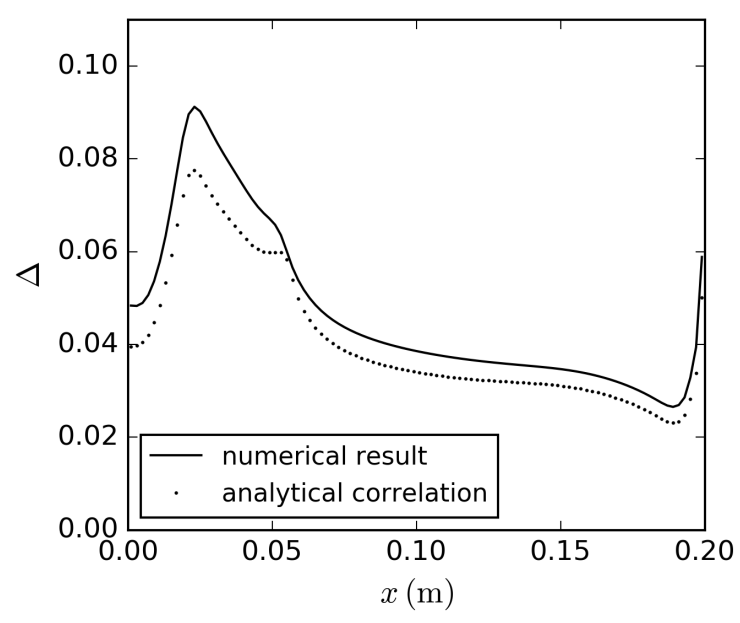

(b) iron

Figure 20: Numerical and analytically predicted convecto-diffusive parameter profiles for aluminum and iron, for a solid fraction $f_{S}=0.44$

\section{Conclusion}

The present study is dedicated to the formulation of wall functions to describe solute boundary layers occurring in directional solidification processes. These wall functions are obtained from a scaling analysis procedure proposed by Garandet et al. [17]. In the present work, specific attention is paid to the description of the turbulent transport for high convective regimes. This point appears relevant since various stirring techniques can be used to enhance solute segregation, leading to turbulent flows in the melt. The influence of the turbulent fluctuations on solute transport is introduced in the scaling analysis using an analytical model. The solute wall functions are compared to the segregation numerical results for a canonical 2D lid driven cavity configuration. This simple and well studied configuration is used as a reference test case to benefit from previous works on the subject. The results are, however, expected to hold for a variety of growth configurations and convective driving forces. Good agreement is found between the analytical and numerical results, especially for an order of magnitude approach.

The developed solute wall functions are then used to compute segregation fields from hydrodynamic simulations. Our aim is to demonstrate that the analytical model can provide meaningful information on axial and lateral segregations. An iterative procedure is proposed to compute solute incorporation in the solid from wall shearstress profiles at the solid/liquid interface. This procedure is applied to the $2 \mathrm{D}$ lid driven cavity configuration 
and analytical and numerical concentration fields are compared. Mean and local errors on analytical concentration fields appear more than reasonable for turbulent flow configurations, even when hydrodynamic simulations are performed without specific interface refinement. These results confirm the interest of the developed method to estimate segregations without a complete numerical resolution of the solute boundary layer. Limitations of the model regarding laminar flows, separation points and impingement points are also discussed.

In our opinion, the analytical model could be applied to industrial configurations where 3D transient segregation simulations require too heavy computational means to allow parametric studies. First information on the solute repartition in the solid could be obtained from a solidification simulation without solute transport and with a relatively coarse mesh at the interface. Nevertheless, the transposition of the procedure to a 3D case with a curved solid/liquid interface is not immediate. A first issue is the analysis of the 3D fluid flow near the interface to extract vortices characteristic lengths. Suitable hydrodynamic wall functions should also be carefully defined regarding the nature of the fluid flow. This point is of primary importance for an accurate estimation of the wall shear-stress at the interface. Besides, in order to enhance the analytical results, specific correlations for the characteristic points could be developed on the basis of former works on radial segregation [37, 38].

Finally, a correlation is proposed to transpose the segregation parameters of a given chemical species to other species. This correlation is directly derived from the analytical model of the solute boundary layer. It can be applied to numerical or experimental results in order to simplify parametric studies. Regarding the application to experimental data, the accuracy of the method is limited by the precision of the known molecular diffusivities. The measurement of these coefficients remains difficult and uncertainties can be significant [35].

\section{Acknowledgement}

The authors are indebted to the institute Carnot Ingnierie@Lyon for its support and funding.

\section{References}

[1] J.R. Davis, A. Rohatgi, R.H. Hopkins, P.D. Blais, P. Rai-Choudhury, J.R. McCormick, and H.C. Mollenkopf. Impurities in silicon solar cells. IEEE Transactions on Electron Devices, 27:677-687, 1980. ISSN 0018-9383. DOI 10.1109/T-ED.1980.19922.

[2] J. Hofstetter, J.F. Lelièvre, C. del Canizo, and A. Luque. Acceptable contamination levels in solar grade silicon: From feedstock to solar cell. Materials Science and Engineering: B, 159-160:299-304, 2009. ISSN 09215107. DOI 10.1016/j.mseb.2008.05.021.

[3] Y. Delannoy. Purification of silicon for photovoltaic applications. Journal of Crystal Growth, 360:61-67, 2012. ISSN 00220248. DOI 10.1016/j.jcrysgro.2011.12.006.

[4] C. Reimann, M. Trempa, T. Jung, J. Friedrich, and G. Müller. Modeling of incorporation of O, N, C and formation of related precipitates during directional solidification of silicon under consideration of variable processing parameters. Journal of Crystal Growth, 312:878-885, 2010. ISSN 00220248. DOI 10.1016/j.jcrysgro.2009.11.070.

[5] K. Dadzis, D. Vizman, and J. Friedrich. Unsteady coupled 3D calculations of melt flow, interface shape, and species transport for directional solidification of silicon in a traveling magnetic field. Journal of Crystal Growth, 367:77-87, 2013. ISSN 00220248. DOI 10.1016/j.jcrysgro.2012.12.135.

[6] C. Stelian and T. Duffar. Numerical modeling of CdTe crystallization from Te solution under terrestrial and microgravity conditions. Journal of Crystal Growth, 400:67-75, 2014. ISSN 00220248. DOI 10.1016/j.jcrysgro.2014.05.001.

[7] Y. Delannoy, F. Barvinschi, and T. Duffar. 3D dynamic mesh numerical model for multi-crystalline silicon furnaces. Journal of Crystal Growth, 303:170-174, 2007. ISSN 00220248. DOI 10.1016/j.jcrysgro.2006.12.075.

[8] Y.Y. Teng, J.C. Chen, C.W. Lu, and C.Y. Chen. The carbon distribution in multicrystalline silicon ingots grown using the directional solidification process. Journal of Crystal Growth, 312:1282-1290, 2010. ISSN 00220248. DOI 10.1016/j.jcrysgro.2009.11.020.

[9] M.P. Bellmann, B. Panjwani, M. Syvertsen, and E.A. Meese. Dynamic simulation of impurity transport and chemical reactions in a Bridgman furnace for directional solidification of multi-crystalline silicon. Journal of Crystal Growth, 369:47-54, 2013. ISSN 00220248. DOI 10.1016/j.jcrysgro.2013.02.008. 
[10] W. Dridi, D. Henry, and H. Ben Hadid. Influence of acoustic streaming on the stability of melt flows in horizontal Bridgman configurations. Journal of Crystal Growth, 310:1546-1551, 2008. ISSN 00220248. DOI 10.1016/j.jcrysgro.2007.11.014.

[11] S. Dumitrica, D. Vizman, J.P. Garandet, and A. Popescu. Numerical studies on a type of mechanical stirring in directional solidification method of multicrystalline silicon for photovoltaic applications. Journal of Crystal Growth, 360:76-80, 2012. ISSN 00220248. DOI 10.1016/j.jcrysgro.2012.01.011.

[12] B. Moudjed, V. Botton, D. Henry, H. Ben Hadid, and J.P. Garandet. Scaling and dimensional analysis of acoustic streaming jets. Physics of Fluids, 26:093602, 2014. ISSN 1070-6631, 1089-7666. DOI 10.1063/1.4895518.

[13] M. Cablea, K. Zaidat, A. Gagnoud, A. Nouri, and Y. Delannoy. Directional solidification of silicon under the influence of travelling magnetic field. Journal of Crystal Growth, 401:883-887, 2014. ISSN 00220248. DOI 10.1016/j.jcrysgro.2013.11.062.

[14] R.A. Negrila, A. Popescu, and D. Vizman. Numerical and experimental modeling of melt flow in a directional solidification configuration under the combined influence of electrical current and magnetic field. European Journal of Mechanics - B/Fluids, 52:147-159, 2015. ISSN 09977546. DOI 10.1016/j.euromechflu.2015.03.001.

[15] J.A. Burton, R.C. Prim, and W.P. Slichter. The distribution of solute in crystals grown from the melt. Part I. Theoretical. The Journal of Chemical Physics, 21:1987-1991, 1953. DOI 10.1063/1.1698728.

[16] L.O. Wilson. On interpreting a quantity in the Burton, Prim and Slichter equation as a diffusion boundary layer thickness. Journal of Crystal Growth, 44:247-250, 1978. ISSN 00220248. DOI 10.1016/0022-0248(78)90199-9.

[17] J.P. Garandet, N. Kaupp, D. Pelletier, and Y. Delannoy. Solute segregation in a lid driven cavity: Effect of the flow on the boundary layer thickness and solute segregation. Journal of Crystal Growth, 340:149-155, 2012. ISSN 00220248. DOI 10.1016/j.jcrysgro.2011.11.071.

[18] S. Kaddeche, J.P. Garandet, D. Henry, H. Ben Hadid, and A. Mojtabi. On the effect of natural convection on solute segregation in the horizontal Bridgman configuration: Convergence of a theoretical model with numerical and experimental data. Journal of Crystal Growth, 409:89-94, 2014. ISSN 00220248. DOI 10.1016/j.jcrysgro.2014.10.009.

[19] M. Chatelain, M. Albaric, D. Pelletier, and V. Botton. Solute segregation in directional solidification: Scaling analysis of the solute boundary layer coupled with transient hydrodynamic simulations. Journal of Crystal Growth, 430:138-147, 2015. ISSN 00220248. DOI 10.1016/j.jcrysgro.2015.08.013.

[20] J.P. Garandet, T. Duffar, and J.J. Favier. On the scaling analysis of the solute boundary layer in idealized growth configurations. Journal of crystal growth, 106:437-444, 1990. DOI 10.1016/0022-0248(90)90090-8.

[21] A. Bejan. Convection Heat Transfer 4e. John Wiley \& Sons, Inc., Hoboken, NJ, USA, 2013. DOI 10.1002/9781118671627.

[22] J. Altenberend, Y. Delannoy, A. Nehari, G. Chichignoud, and K. Zaidat. Modelling and experiments for the convecto-diffusive removal of impurities from the solidification front. Journal of Crystal Growth, 458:80-86, 2017. ISSN 00220248. DOI 10.1016/j.jcrysgro.2016.11.052.

[23] D.B. Spalding. Concentration fluctuations in a round turbulent free jet. Chemical Engineering Science, 26: 95-107, 1971. ISSN 00092509. DOI 10.1016/0009-2509(71)86083-9.

[24] Y. Tominaga and T. Stathopoulos. Turbulent Schmidt numbers for CFD analysis with various types of flowfield. Atmospheric Environment, 41:8091-8099, 2007. ISSN 13522310. DOI 10.1016/j.atmosenv.2007.06.054.

[25] K. Koeltzsch. The height dependence of the turbulent Schmidt number within the boundary layer. Atmospheric Environment, 34:1147-1151, 2000. ISSN 13522310. DOI 10.1016/S1352-2310(99)00369-6.

[26] D.P. Combest, P.A. Ramachandran, and M.P. Dudukovic. On the Gradient Diffusion Hypothesis and Passive Scalar Transport in Turbulent Flows. Industrial \& Engineering Chemistry Research, 50:8817-8823, 2011. ISSN 0888-5885, 1520-5045. DOI 10.1021/ie200055s. 
[27] A.M. Bianchi, Y. Fautrelle, J. Etay, and R. Longchamp. Transferts thermiques. Presses Polytechniques et Universitaires Romandes, Lausanne, 2004. ISBN 2-88074-496-2.

[28] ANSYS Inc. Ansys fluent 14.0 - theory guide, 2011.

[29] Y. Delannoy and K. Zaidat. Modelling the segregation of impurities during solidification with turbulent electromagnetic stirring. In The 7 th International Conference on Electromagnetic Processing of Materials, pages 217-220, 2012.

[30] F.B Santara. Cristallisation du silicium photovoltaïque sous induction électromagnétique: étude d'une vanne de rétention et de la ségrégation sous brassage. Ph. D. Thesis, Institut National Polytechnique de Grenoble-INPG, 2010.

[31] J.P. Garandet, S. Corre, S. Kaddeche, and T. Alboussière. The influence of convection on the duration of the initial solute transient in alloy crystal growth. Journal of Crystal Growth, 209:970-982, 2000. ISSN 00220248. DOI 10.1016/S0022-0248(99)00630-2.

[32] J.P. Garandet. Microsegregation in crystal growth from the melt: an analytical approach. Journal of Crystal Growth, 131:431-438, 1993. ISSN 00220248. DOI 10.1016/0022-0248(93)90192-Y.

[33] H. Kodera. Diffusion Coefficients of Impurities in Silicon Melt. Japanese Journal of Applied Physics, 2:212-219, 1963. ISSN 0021-4922, 1347-4065. DOI 10.1143/JJAP.2.212.

[34] D. Morvan, J. Amouroux, and G. Revel. Mise au point d'une technique de fusion de zone sous plasma appliquée à la préparation du silicium photovoltaïque. Revue de Physique Appliquée, 15:1229-1238, 1980. ISSN 0035-1687. DOI 10.1051/rphysap:019800015070122900.

[35] J.P. Garandet. New Determinations of Diffusion Coefficients for Various Dopants in Liquid Silicon. International Journal of Thermophysics, 28:1285-1303, 2007. ISSN 0195-928X, 1572-9567. DOI 10.1007/s10765-0070205-z.

[36] K. Tang, E.J. Øvrelid, G. Tranell, and M. Tangstad. Critical assessment of the impurity diffusivities in solid and liquid silicon. JOM, 61:49-55, 2009. ISSN 1047-4838, 1543-1851. DOI 10.1007/s11837-009-0167-7.

[37] J.P. Garandet, N. Kaupp, and D. Pelletier. The effect of lid driven convective transport on lateral solute segregation in the vicinity of a crucible wall. Journal of Crystal Growth, 361:195-200, 2012. ISSN 00220248. DOI 10.1016/j.jcrysgro.2012.09.026.

[38] J.P. Garandet. On the problem of radial segregation in an idealized horizontal Bridgman configuration: scaling and numerical approaches. Journal of crystal growth, 114:593-602, 1991. DOI 10.1016/0022-0248(91)90405-T. 